\title{
In a Temporally Segmented Experience Hippocampal Neurons Represent Temporally Drifting Context But Not Discrete Segments
}

\author{
○John H. Bladon, ${ }^{1,2} \odot$ Daniel Joseph Sheehan, ${ }^{1}$ Camila S. De Freitas, ${ }^{1}$ and ${ }^{\circledR}$ Marc W. Howard ${ }^{1}$ \\ ${ }^{1}$ Center for Memory and Brain, and ${ }^{2}$ Graduate Program for Neuroscience, Boston University, Boston, Massachusetts 02215
}

There is widespread agreement that episodic memory is organized into a timeline of past experiences. Recent work suggests that the hippocampus may parse the flow of experience into discrete episodes separated by event boundaries. A complementary body of work suggests that context changes gradually as experience unfolds. We recorded from hippocampal neurons as male Long-Evans rats performed 6 blocks of an object discrimination task in sets of 15 trials. Each block was separated by removal from the testing chamber for a delay to enable segmentation. The reward contingency reversed from one block to the next to incentivize segmentation. We expected animals to hold two distinct, recurring representations of context to match the two distinct rule contingencies. Instead, we found that overtrained rats began each block neither above nor below chance but by guessing randomly. While many units had clear firing fields selective to the conjunction of objects in places, a significant population also reflected a continuously drifting code both within block and across blocks. Despite clear boundaries between blocks, we saw no neural evidence for event segmentation in this experiment. Rather, the hippocampal ensemble drifted continuously across time. This continuous drift in the neural representation was consistent with the lack of segmentation observed in behavior.

Key words: electrophysiology; episodic memory; event segmentation; hippocampus; rat; temporal context

\section{Significance Statement}

The neuroscience literature yet to reach consensus on how the hippocampus supports the organization of events across time in episodic memory. Initial studies reported stable hippocampal maps segmented by remapping events. However, it remains unclear whether segmentation is an artifact of cue responsivity. Recently, research has shown that the hippocampal code exhibits continuous drift. Drift may represent a continually evolving context; however, it is unclear whether this is an artifact of changing experiences. We recorded dCA1 in rats performing an object discrimination task designed to segment time. Overtrained rats could not anticipate upcoming context switches but used context boundaries to their advantage. Hippocampal ensembles showed neither evidence of alternating between stable contexts nor sensitivity to boundaries, but showed robust temporal drift.

\section{Introduction}

Episodic memory refers to the recollection of a specific event situated in a unique place and time (Tulving and Madigan, 1970). In his description of episodic memory, Tulving emphasized that a difference between episodic and semantic memory is that episodic memories are temporally dated or are remembered in rela-

\footnotetext{
Received June 4, 2018; revised June 17, 2019; accepted June 20, 2019.

Author contributions: J.H.B. and D.J.S. designed research; J.H.B., D.J.S., and C.S.D.F. performed research; J.H.B. contributed unpublished reagents/analytic tools; J.H.B., D.J.S., C.S.D.F., and M.W.H. analyzed data; J.H.B. wrote the first draft of the paper; J.H.B., D.J.S., and M.W.H. edited the paper; J.H.B. and M.W.H. wrote the paper.

This project was originally conceived and designed in part by Dr. Howard Eichenbaum. Sadly, he passed away before the data were fully analyzed and therefore was unable to evaluate this manuscript in final form. The authors gratefully acknowledge his many contributions to this experiment, as well as the inspiration we have derived from his leadership. We also thank Dr. Chris Keene for performing the initial pilot work for this experiment; and Dr. Michael Hasselmo for the close support and engaging discussions concerning this manuscript.

The authors declare no competing financial interests.
}

tion to other events across time. The hippocampus is essential for episodic memory and is thought to mediate this function by binding events to a representation of spatial and temporal context (Tulving, 1972; Eichenbaum et al., 2007). Hippocampal ensembles are theorized to represent a "cognitive map" (O'Keefe and Nadel, 1978) that acts as a contextual or relational scaffold onto which events may be bound together for later retrieval ( $\mathrm{Da}$ vachi, 2006).

There are two complementary models for how spatiotemporal context is structured in the hippocampus. Event segmentation theory suggests that experience is segmented across time into discrete situational contexts (see Fig. 1, Event Segmentation)

Correspondence should be addressed to John H. Bladon at jhbladon@bu.edu.

https://doi.org/10.1523/JNEUROSCI.1420-18.2019

Copyright $\odot 2019$ the authors 
(Muller and Kubie, 1987; Zacks et al., 2001; Baldassano et al., 2017; DuBrow et al., 2017). Temporal context theory suggests that the hippocampal representation of context evolves continually (see Fig. 1, Temporal Context) (Howard et al., 2005; Howard and Eichenbaum, 2013).

Consistent with event segmentation theory, behavioral and neuroimaging evidence suggests that experiences are organized by discrete situational contexts (Muller and Kubie, 1987; Zacks et al., 2001; Baldassano et al., 2017; DuBrow et al., 2017). Abrupt changes in environmental or contextual cues across time can cause a behavioral separation in memory traces (Zacks et al., 2001; Ezzyat and Davachi, 2011; Sols et al., 2017). Hippocampal BOLD activity in humans, and ensemble activity in rodents increases when a border between contexts is perceived, as if the hippocampus parcellates experience into contextual chunks (Mack et al., 2016; Place et al., 2016; Baldassano et al., 2017; DuBrow et al., 2017; Bulkin et al., 2018). Hippocampal place cells generate separate maps across different spatial environments and across a variety of explicit contextual designations that occur in the same physical space (Markus et al., 1994; Kobayashi et al., 1997; Leutgeb et al., 2005; Wills et al., 2005; Komorowski et al., 2009; Brandon et al., 2014; Smith and Bulkin, 2014). In a particularly clear example, neural segmentation across two physical contexts developed as rats learned to discriminate between the two contexts (Komorowski et al., 2009, 2013).

Consistent with temporal context theory, the contiguity effect describes the tendency for subjects to bind together unrelated events that occurred together in time, and has been shown across timescales in neural and behavioral datasets (Kahana, 1996; Zaromb et al., 2006; Howard et al., 2008; Manning et al., 2011; Folkerts et al., 2018). In humans, hippocampal BOLD signals during recall of events reflect a broad continuum of relatedness that maps onto the temporal and spatial proximity of those events (Schapiro et al., 2012, 2016; Hsieh et al., 2014; Nielson et al., 2015; Deuker et al., 2016; Jenkins and Ranganath, 2016). A large body of research reports slow changes in the hippocampal representation of place across extended time (Manns et al., 2007; Paz et al., 2010; Mankin et al., 2012, 2015; Ziv et al., 2013; Rubin et al., 2015; Folkerts et al., 2018; Mau et al., 2018). Crucially, these studies report a spectrum of stability levels such that there are both drifting and stable components of the neural code (Ziv et al., 2013; Rubin et al., 2015; Mau et al., 2018).

There is behavioral, neuroimaging, and animal neurophysiology evidence consistent with both temporal drift and event segmentation, and the two hypotheses are not mutually exclusive (see Fig. 1, Segmented Temporal Context). However, these two bodies of work have never been directly compared in the same preparation.

\section{Materials and Methods}

Subjects. Subjects were 4 male Long-Evans rats (Charles River) weighing between 350 and $450 \mathrm{~g}$ and between the ages of 6 months and 1 year for the duration of the experiment. All animals were single housed and maintained on a $12 \mathrm{~h}$ light/dark cycle (lights on 8:00 A.M. to 8:00 P.M.). Behavioral training and testing were conducted exclusively during the light phase. Animals were maintained at a minimum (85\%) of their ad libitum feeding body weight during all behavioral training and testing periods. Procedures were conducted in accordance with the requirements set by the National Institutes of Health and Boston University Institutional Animal Care and Use Committee.

Behavioral apparatus. The behavioral training and testing environment was a custom-built wood apparatus $(401 \times 60 \mathrm{w} \times 40 \mathrm{~h} \mathrm{~cm})$ consisting of a $40 \mathrm{~cm} \times 40 \mathrm{~cm}$ box and a $20 \mathrm{~cm} \times 20 \mathrm{~cm}$ side alleyway. The objects consisted of identical circular terra cotta pots $(10 \mathrm{~cm}$ high with an internal diameter of $9 \mathrm{~cm}$ ), each with their own unique digging media and odors (e.g., purple beads with grapefruit scent). The pots were distinguishable only by their scent and digging media, requiring the animal to overtly sample before choosing to dig. To prevent the animals from being guided by odor of the Froot Loop (Kellogg's) cereal reward, finely crushed Froot Loops were sprinkled into all digging media.

Surgery. Anesthesia was induced by inhalation of 5\% isoflurane (Webster Veterinary Supply) in oxygen, and then a stable plane was maintained at $1.5 \%-3 \%$ throughout the entirety of surgery. Before surgery, animals were injected with the analgesic Buprenex (buprenorphine hydrochloride, $0.03 \mathrm{mg} / \mathrm{kg}$ i.m.; Reckitt Benckiser Healthcare) and the antibiotic cefazolin $(330 \mathrm{mg} / \mathrm{ml}$ i.m.; West-Ward Pharmaceutical). The skin of the animal's head covering the skull was shaved and cleaned with alcohol swabs before then being placed in a stereotaxic frame (Kopf Instruments). A longitudinal incision was made to expose the skull and the bone, and underlying fascia was cleared to gain access to stereotaxic coordinates and locations for anchoring screws. Animals were implanted with microdrives containing 18-24 independently drivable tetrodes targeting the dorsal pole of the CA1 cell layer of the hippocampus (centered at $3.6 \mathrm{~mm}$ posterior and $2.6 \mathrm{~mm}$ lateral from bregma). Finally, a screw was placed above the cerebellum to serve as a ground signal. Each tetrode was composed of four $12 \mu \mathrm{m}$ RO 800 wires (Sandvik Kanthal HP Reid Precision Fine Tetrode Wire, Sandvik). Tetrodes were plated with noncyanide gold solution, via electrolysis to reduce impedance to between 180 and $220 \mathrm{k} \Omega$. At the conclusion of the surgery, all tetrodes were gradually lowered $\sim 0.5-1.5 \mathrm{~mm}$ into tissue. Upon recovery from anesthesia, animals underwent postoperative care for $3 \mathrm{~d}$ and received doses of Buprenex and cefazolin, as described above, two times a day $(12 \mathrm{~h}$ intervals). Animals were allowed to recover 1 week before behavioral testing commenced.

Neural recordings. Electrophysiological recordings for this project were collected on a 96 channel OmniPlex D Neural Acquisition System (Plexon). Each channel was amplified on head-mounted preamps and then amplified again for total of $1000 \times$ to $10,000 \times$ before being digitized at $40 \mathrm{kHz}$. Spike data were bandpass filtered from 200 to $8.8 \mathrm{kHz}$ and local field potentials from 1.5 to $400 \mathrm{~Hz}$. Spike channels were referenced to a local electrode in the same region to remove both movement-related and ambient electrical noise. That local reference electrode was then referenced to ground and provided the local field potential signal in the region. Action potentials of neurons were detected via threshold crossing and then sorted later using Offline Sorter (Plexon). Only units collected on tritrodes or tetrodes were sorted, and all clusters with $>0.1 \%$ interspike interval violations of within 2 ms were removed. Cineplex Studio (Plexon) was used for capturing behavioral tracking data, and Cineplex Editor (Plexon) was used to enter event markers and to verify animal tracking data. Between recorded training sessions, tetrodes were advanced at a minimum of $40 \mu \mathrm{m}$ and positioned based on visual inspection of spike clusters to maximize neural unit yield. Tetrodes were allowed to settle after turning over a period of days to prevent contamination of neural signals with tetrode drift.

Histology. Upon completion of behavioral testing, rats were anesthetized with $<5 \%$ isoflurane in oxygen. Anatomical recording sites were confirmed by creating a small lesion in the brain tissue by passing a $40 \mu \mathrm{A}$ current until the connection was severed (generally $2-8 \mathrm{~s}$ ). Immediately after completion of electrolytic lesions, animals received an overdose injection (interperitoneal) of Euthasol (Virbac AH) and upon cessation of breathing were immediately transcardially perfused with ice-cold $0.5 \%$ potassium PBS followed by $5 \%$ phosphate buffered formalin (VWR). Brains were then removed and placed in additional $5 \%$ formalin phosphate for at least $36 \mathrm{~h}$. Brains were then submerged in 30\% sucrose for cryoprotection until sectioning into $40-\mu \mathrm{m}$-thick sections via cryostat (CM 3050s; Leica Biosystems). Brain sections were processed using standard Nissl staining protocol to visually confirm tetrode-recording sites (see Fig. 1C).

Experimental design and statistical analysis. To determine how the hippocampal map segments similar experiences that occur across minutes, rats performed a task in which distinct behaviors were reinforced in different temporally blocked chunks of trials in the same spatial environment. The boundary between blocks was cued by shuttling the animal to 
a separate chamber for $1 \mathrm{~min}$, but there were no overt cues to signal the behavioral context at the time of the choice behavior. A representation of temporal context that changes continuously across blocks would be behaviorally suboptimal in this experiment (see Fig. 1, Drifting Temporal Context). Rather, the strategy to maximize reward would be to segment the experiment into behaviorally meaningful contexts by using the boundary cue (see Fig. 1, Event Segmentation).

During performance of this task, we recorded extracellularly from dorsal CA1 ensembles. As in earlier work that showed evidence for event segmentation across space (Komorowski et al., 2009, 2013), rats were presented with pseudorandomly positioned pairs of pots containing unique odors and digging media, and the rat was rewarded for choosing the correct pot from the pair. The identity of the rewarded pot was consistent for each block of 15 trials after which the 1 min boundary cue was imposed; for the next 15 trials, the other pot was rewarded. The event segmentation hypothesis predicts two stable mappings of objects and places: one for each rule condition (see Fig. 1, Event Segmentation). In contrast, the temporal context hypothesis predicts a continuous decorrelation of neural representations that was unaffected by the blocked structure of the experience (see Fig. 1, Drifting Temporal Context). A representation validating both theories might involve a new but stable context instated at the start of each block of trials (see Fig. 1, Segmented Temporal Context).

Animal training and task. Once each animal recovered from surgery, they were initially trained to dig for Kellogg's Froot Loop bits in an aloe-scented pot over a cloves-scented pot (both in sand). Once they reliably dug in the aloe pot and refrained from digging in the cloves pot, training began in the blocked-reversal task. Each training and recording session consisted of 6 blocks of 15 trials, for a total of 90 trials. Once the rat reached a criterion of $70 \%$ correct across a given session, recording commenced. Some sessions were terminated early (the shortest session was 85 trials) due to lack of motivation by the subject. Within each 15 trial block, the reward contingency was set so that one pot always had food and the other pot did not. Each trial started with the insertion of a divider so that the experimenter could place the pots pseudorandomly in one of the two positions. The orientation of the two pots was random in each trial, with the exception that a configuration could not be repeated more than three consecutive times. Once pots were placed, the divider was removed and the animal was allowed to sample each pot, but only allowed to dig in one. Upon digging, the unchosen pot was immediately removed and the animal was allowed to dig until he found reward or gave up because he chose incorrectly. After either completion of reward consumption or a $3 \mathrm{~s}$ delay following pot removal, the rat was shuttled to the far half of the chamber and the divider replaced. The next trial commenced immediately until trial 15 was reached. After the last trial of each block, the rat was shuttled into the side alley to wait for $60 \mathrm{~s}$. After the break, the reward contingency was reversed and the next block of 15 trials was performed.

Quantitative and statistical analysis. All analyses of the collected data were performed using custom scripts from MATLAB (The MathWorks). ANOVAs were performed using the "anovan" function in MATLAB under a standard Type 2 sum of squares. For individual unit analyses, perievent histograms were generated from 120 to $160 \mathrm{~ms}$ bins and smoothed using a moving average of a three bin span. All trials were included in perievent rasters, including those in which the rat responded incorrectly, but only the first sample in each trial is shown. Right and left samples correspond to each of the two pseudorandomized positions of the reward pots. Error bars on firing rates were calculated using the SEM. All spatial firing rate plots were generated using a $3 \mathrm{~cm}$ pixel size, and then convolved with a Gaussian smoothing kernel with a SD of one pixel. Selectivity was evaluated by calculating a selectivity score below, where $n$ represents the set of trial types ( 2 in the case of object and position, 4 in the case of object by position), and $\lambda$ represents the mean firing rate for that event type. $\lambda$ pref represents the trial type with the largest firing rate as follows:

Selective Index $=\frac{\left(n-\sum_{i=1}^{n}\left(\frac{\lambda_{i}}{\lambda_{\text {pref }}}\right)\right)}{n-1}$
Significance was determined by generating a null distribution of selectivity scores after randomizing the trial identity of each sample. Only units that passed a $5 \%$ significance threshold from 1000 boots were considered to code a particular dimension (Keene et al., 2016).

For waveform and spike rate drift metrics, average waveform amplitude and spike rate were estimated for each $10 \mathrm{~s}$ bin spanning the whole recording session. We then measured the absolute Euclidean distance of the average waveform shape and absolute difference in firing rate between each bin to all other bins. From that matrix, we regressed the values as a function of distance from the diagonal but excluding the diagonal to obtain an average waveform or spike rate distance as a function of temporal lag between bins for each cell. Unit cluster quality was analyzed using standard $\mathrm{L}$ ratio and isolation distance metrics (Schmitzer-Torbert et al., 2005). In short, $\mathrm{L}$ ratio and isolation distances were calculated for each unit compared with all other clustered units from the 12 dimensions (spike amplitude, peak-valley, and energy for each wire) that were used during manual sorting. Units that exceeded the 95th percentile L ratio (0.013) or were below the 5 th percentile of isolation distance (6.57) were considered poor clusters and removed from all population analysis. Units with an average firing rate of $>2 \mathrm{~Hz}$ and a waveform half-width of $<0.16 \mathrm{~ms}$ across the whole recording session were assumed to be interneurons and were also removed from all population analyses (see Fig. 7).

For population analyses, trial rate vectors were constructed for each cell by averaging the firing rate across the $2 \mathrm{~s}$ ( 1 second before to $1 \mathrm{~s}$ after) surrounding the first sampling event on each trial, and then $z$-normalizing the rate across trials for each individual neuron. All trials were included in these analyses unless otherwise noted so as to reveal any performance effects as well as for statistical reasons. A $z$ transform was used to prevent over-reliance on highly active units or under-reliance on sparsely active units. A population vector correlation matrix was generated for each rat by calculating the Spearman correlation of the population vector for each trial to each other (see Fig. 9B). When trials were aggregated based on their temporal lag instead of trial lag, $20 \mathrm{~s}$ bins were used. That correlation matrix was then averaged across all sessions to generate the "super rat" matrix observed in Figure 9A. All measures of drift were constructed by first generating a mean value for each session onto which statistics were performed, and the mean \pm SEM across sessions was plotted. Bootstrap permutation tests were performed as described with a standard 10,000 randomized samples in which the group index was randomized for each rat without replacement. Bayes factors were obtained by inputting summary statistics into an online engine accessible at http://pcl.missouri.edu/ (Liang et al., 2008).

\section{Results}

\section{Rats performed a temporally blocked object} discrimination task

Rats were trained to perform a blocked object discrimination task designed to segment memory into six 15 trial blocks (Fig. 1A). Within each block of 15 trials, one of the two distinguishable pots contained hidden reward. After each 15 trial block, a temporal delay signaled the end of a block; the reward contingency was reversed to the other pot for the subsequent block of trials (Fig. 1, Event Segmentation). Between blocks, the rat was shuttled into a side chamber for $60 \mathrm{~s}$ so that the end of a block was signaled not only by an increased trial duration per se but also by an intervening experience. Individual trials took on average a little $<30$ s to perform $(25.4 \pm 0.39 \mathrm{~s})$, each block of trials took $\sim 6 \mathrm{~min}(5.92 \pm$ $0.1 \mathrm{~min})$, and a session lasted $\sim 45 \mathrm{~min}(44.82 \pm 1.1 \mathrm{~min})$. Each session contained an unequal but approximately similar number of sampling events at each item and position; however, some animals showed a slight bias toward sampling the item on one side of the maze more often. Furthermore, we restricted our analyses to the first sampling event on each trial, as the identity and position of the object on the first sample remained pseudorandomized per task design. Only after the sample had terminated and a response was offered did the behavior systematically change 
A
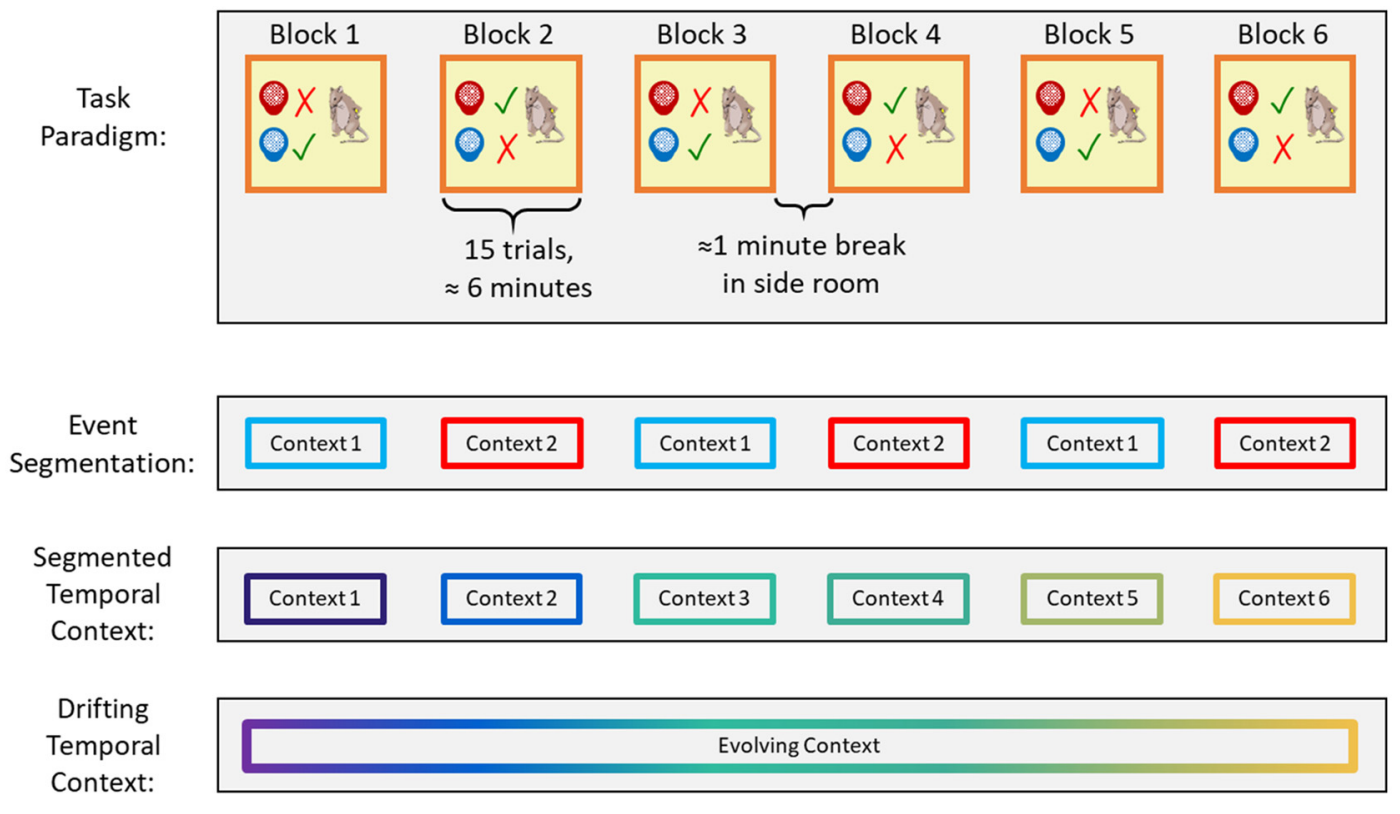

B

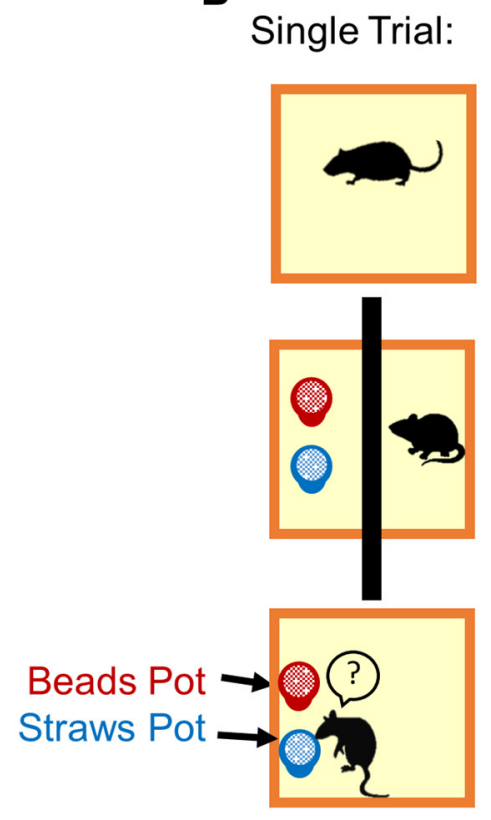

C

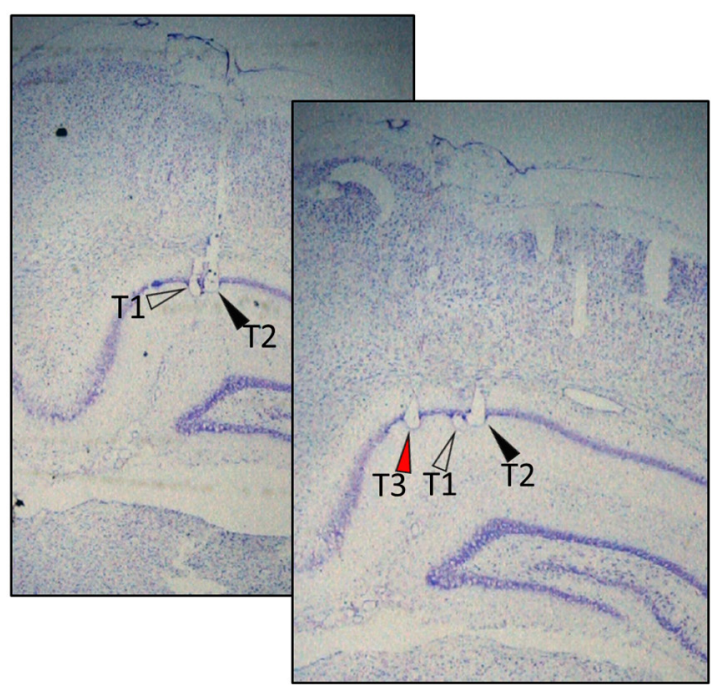

Figure 1. Task design. $A$, Rats performed a blocked object discrimination task in which the reward contingency was held constant for 15 trials in a row and then reversed for the next 15 . The pots were placed pseudo-randomly on each trial in the positions shown above. Each block took $\sim 6 \mathrm{~min}$, and each delay between blocks was fixed at $1 \mathrm{~min}$. $\boldsymbol{B}$, Each trial consisted of three phases: a short intertrial interval, the pot setup phase, and the sample/choice phase. C, Recording locations of three individual tetrodes. Arrowheads indicate final tetrode locations in the pyramidal layer of dorsal CA1.

toward rejecting the incorrect object and digging in the correct pot.

\section{Rats performed as though each block of trials was a new episode}

Following pretraining on simple pot discrimination, rats took $\sim 1$ week to reach a criterion of $70 \%$ correct within a given session. Recording began after criterion was reached. Once trained, all rats performed similarly to each other (mean across rats $=$ $80 \% \pm 1.97 \%$, ANOVA, $\left.F_{(3,19)}=0.4\right)$, and all rats performed similarly across days (mean across days $=79 \% \pm 0.52 \%$, ANOVA, $\left.F_{(7,15)}=0.64\right)$ and blocks within each day (mean across blocks $=79 \pm 1.13 \%$, ANOVA, $\left.F_{(5,115)}=1.72\right)$. Typically, errors were concentrated around the beginning of each block but were not restricted to the beginning of the recording session (Fig. 2). When the rat's spatial trajectory was examined on a trial-to-trial and block-to-block basis, rats tended to approach whichever pot they were closest to on each trial (Fig. 3C,D). However, in a minority of sessions, we observed the rat slowly change its starting position across trials ( 7 of 23 sessions exhibited a significant correlation between starting position and trial start timestamp; $p<0.05$ following a Bonferroni correction). Similarly, after examining each block of trials individually (a total of 138 blocks from 23 sessions), a minority of blocks of trials included stereo- 

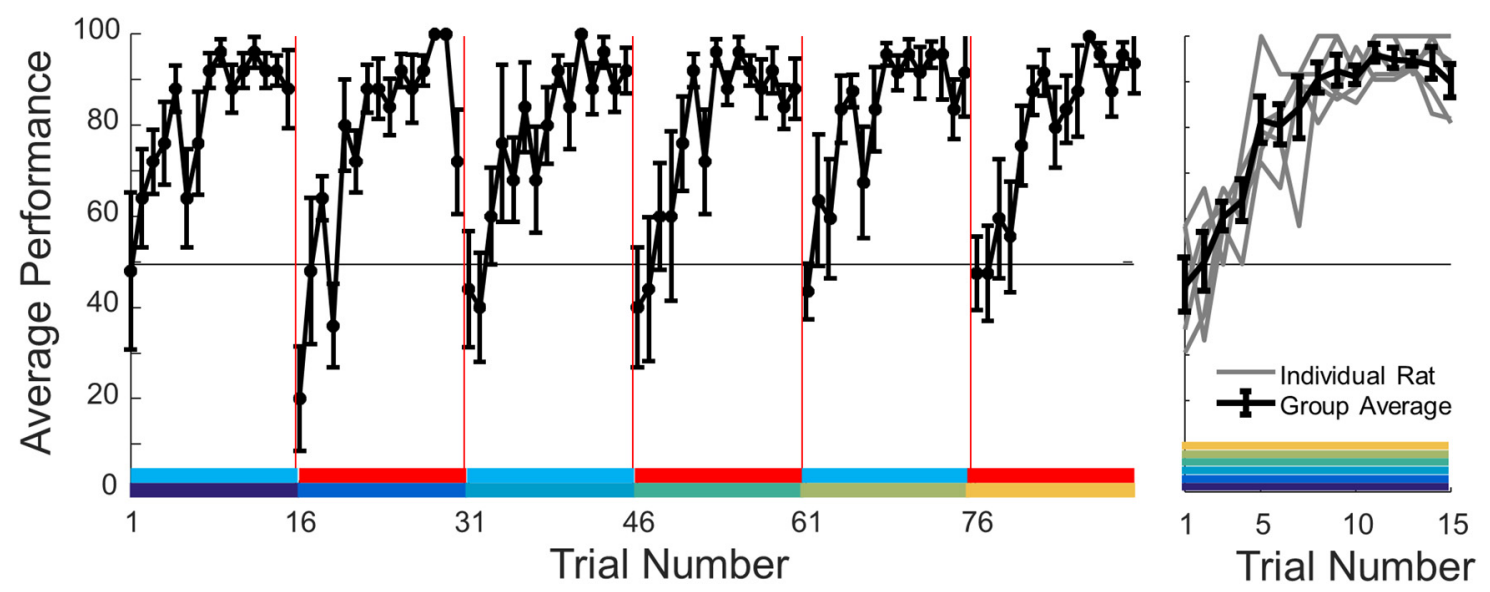

Figure 2. Rats' behavior suggests that they did not anticipate the reversal in reward contingency across blocks. Left, Mean \pm SEM performance throughout each recording session. Gray lines indicate individual rats. Rats began each block in a session at chance regardless of the position of that block in the session. Colored bars represent changing context per Figure 1 . Right, When all blocks were concatenated (colored blocks stacked), no rat performed better or worse than chance at the beginning of each block. Gray lines indicate individual rats. Black line indicates mean across all rats. Red line indicates chance performance. All rats behaved as though each block represented a novel context in which to learn the rule contingency. Colored bars represent evidence toward "temporal context" as denoted in Figure 1.

typed changes in starting position across trials ( 6 blocks of 138 exhibited a significant correlation between starting position and trial start timestamp; $p<0.05$ following a Bonferroni correction). These observations suggest that only a minority of sessions and blocks involved changes in behavior over time. For the vast majority of sessions and blocks of trials, the rat's behavioral sequence did not vary across time. Therefore, overall each animal rapidly and repeatedly learned the rule contingency from chance each block but maintained a stable sequence of movements throughout the session.

We generated two alternative hypotheses regarding behavior. We hypothesized that the rats would recognize the block transitions as a cue to change their response strategy, and would begin each block by switching their response toward digging in the now-correct pot. Alternatively, rats could have ignored the boundary cue. In this case, the animals would begin each block by erroneously perseverating with the (now-incorrect) response from the previous block. We evaluated these hypotheses at the group level, but also at the individual animal level (if some rats respected the contextual cues while others did not, the group could perform at chance on average despite none of the individual rats performing at chance). To investigate both hypotheses, we compared each rat's performance for each trial across blocks to chance by performing a binomial test on each animal. A twosided binomial test asks whether the probability of correct responses was either above or below chance. Contrary to both hypotheses, only 1 rat performed different from chance for either of the first two trials in each block before a Bonferroni correction (Fig. 2; two-sided binomial test, Rat 1 Trial 1: 30\% uncorrected binomial, $p=0.03$, Trial 2: $38 \%$, binomial, $p=0.24$ : other rats Trial 1: $46.0 \pm 6.4 \%$, minimum, $p=0.09$, Trial 2: $46.2 \pm 6.07 \%$, minimum, $p=0.31)$. By Trial 5 , all rats were performing significantly above chance (Fig. 2; Trial 5: $76.8 \pm 2.0 \%$, all binomial, $p<0.05$, Trials 6-15: $89 \pm 2.2 \%$, all binomial, $p<0.001)$. Rats appeared to begin each block by impulsively digging in the first pot they encountered, as the probability of rejecting the first encountered pot on the first trial of each block was lower than for the last 8 trials of each block (Trial 1 reject rate mean $=0.32 \pm$ $0.04 \%$, trials $11: 15$ reject rate mean $=0.43 \pm 0.02 \%$, rank-sum, $p<0.05)$. Thus, rats appeared to begin each block of trials by guessing and then rapidly learning the new rule contingency. The performance of each rat on each block could be described as a recency-weighted averaging over recent experiences. Alternatively, they may have perceived the block delay as a cue indicating a new context, but the behavioral contingency of that context was unknown to the rat. Both hypotheses account for the chance performance following the interblock delay and also the learning of the new reward contingency within block (Fig. 2, right). However, an interpretation consistent with recency-weighted averaging predicts a neural representation consistent with drifting temporal context (Fig. 1), whereas recognition of the boundary, but not anticipation of the rule, predicts a neural representation consistent with either a segmented temporal context or event segmentation (Fig. 1).

\section{Single units replicated prior findings of object and position} selective fields but were impacted by context

We recorded from 757 cells across 23 sessions from 4 rats each implanted with a 24-tetrode hyperdrive aimed at dorsal CA1 (Fig. $1 C)$. Single-unit activity was observed by generating spatial heat plots (Fig. $3 F-L$ ), and perievent rastergrams, and histograms centered on pot-sampling events (Fig. 4). Consistent with previous reports, a large proportion of place cells had firing fields where the objects were presented (Fig. $3 G-I, K$ ). When event-locked firing was examined, there was a large overlapping population of units whose firing fields discriminated between sampling events. Some units had firing fields that consistently discriminated between objects regardless of the objects' position (Fig. 4, top row; 301 cells, or $40 \%$ of putative pyramidal cells showed an object selectivity score $>95 \%$ of 10,000 bootstrap permutations). There were also units whose fields were selective to one object position (Fig. 3; 247 cells, or 33\% of putative pyramidal cells showed a position selectivity score $>95 \%$ of 10,000 bootstrap permutations) as well as a largely overlapping population that was specific to one object in one position (Fig. 4, bottom row; 247 cells, or $33 \%$ of all putative pyramidal cells showed object by position selectivity $>95 \%$ of 10,000 bootstrap permutations). These proportions appeared to be in line with previous observations (McKenzie et al., 2014). However, upon further investigation, firing fields clearly changed when the rule contingency was altered (Fig. 5). 
A

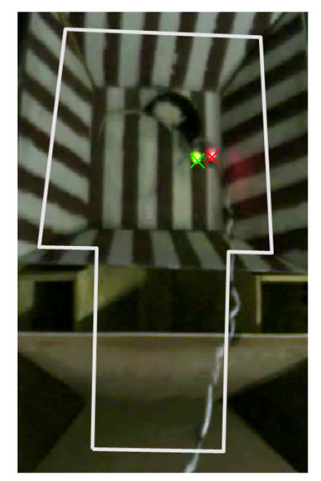

B

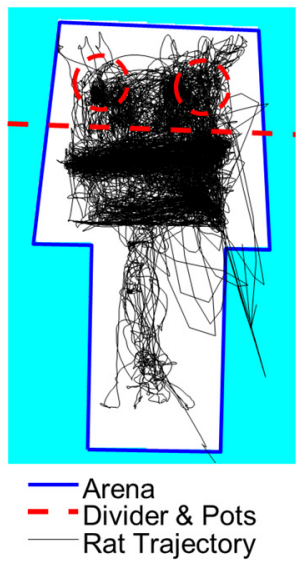

C

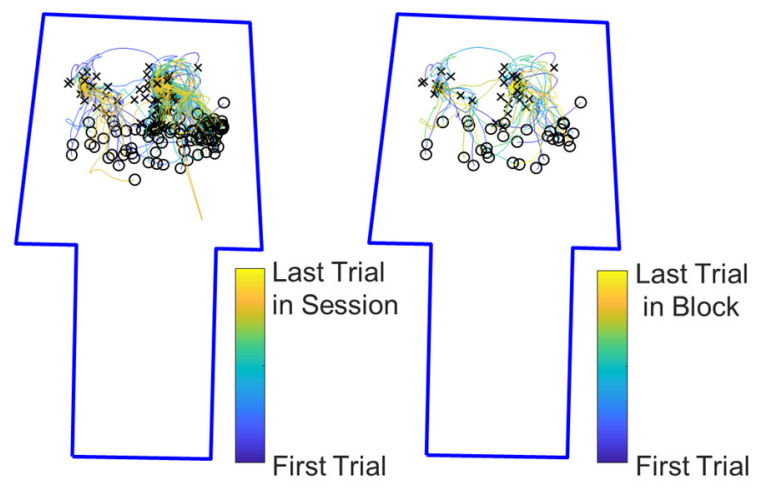

E

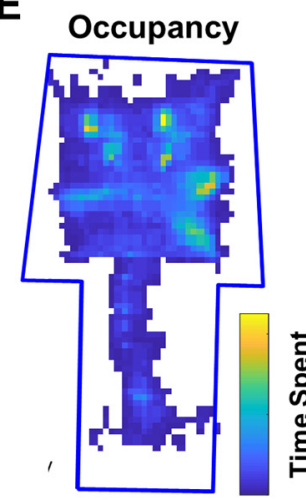

I

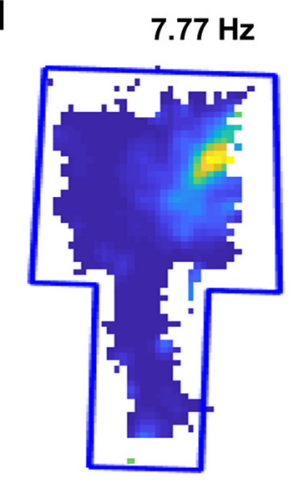

F

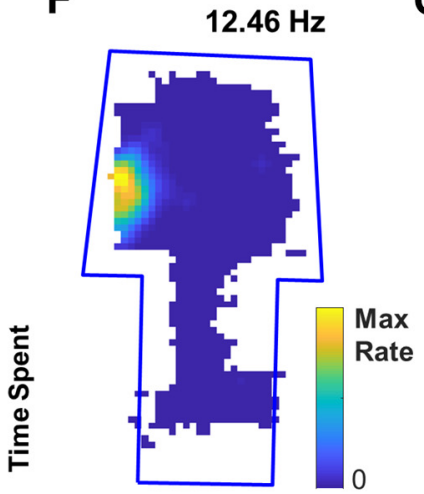

J

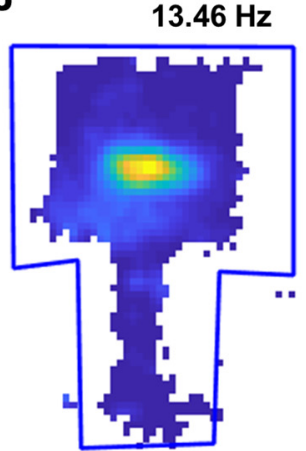

G

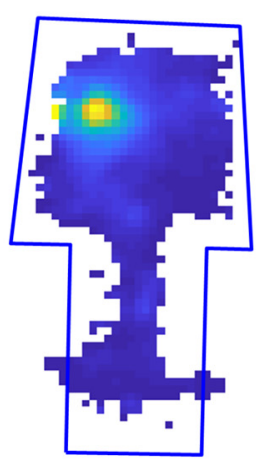

K

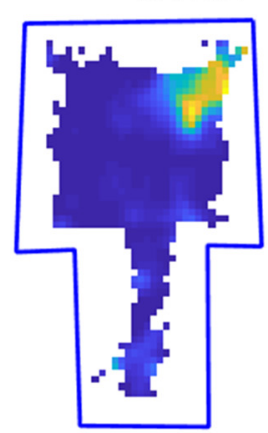

H

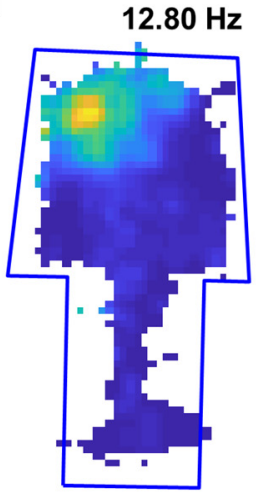

L

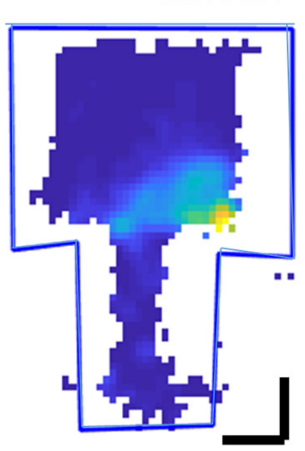

Figure 3. Rats' movements were stereotyped across trials and blocks, and place cells were evident. $\boldsymbol{A}$, Video recording frame illustrating task chamber and rat position. $\boldsymbol{B}$, LED tracking and position of arena, divider, and pots. C, For the vast majority of sessions, the rats' approach to pots was consistent across trials within the session, and (D) within a block. 0 indicates the starting point of each trajectory. X indicates the rats' head position at the first choice. $\boldsymbol{E}$, Example occupancy plot. $\boldsymbol{F}-\boldsymbol{L}$, Spatial heat maps of binned-firing rates for each unit with maximum rate denoted above each plot. Units $\boldsymbol{F}, \boldsymbol{G}$, and $\boldsymbol{H}$ were recorded from Rat 1. Units I and $\boldsymbol{H}$ were recorded from Rat 2. Units $\boldsymbol{J}$ and $\boldsymbol{K}$ were recorded from Rat 4. Calibration: 10 pixels, $30 \mathrm{~cm}$.

A drifting contextual representation replicates previous findings and is uncorrelated to waveform drift

Many units showed firing fields that were modulated by the changing context. The temporal context model and the event segmentation model each make a strong prediction for how context may modulate hippocampal firing fields (Fig. 5). While putative pyramidal cells maintained the same selectivity to object position and identity across blocks of trials, their rates showed obvious changes across contexts that seemed to resemble predictions from the temporal context model (Fig. 5, bottom). Figure 5 contains plots that include all trials, including error trials, and each trial was color-coded based on block (see legend to the right of the figure). Briefly, event segmentation theory predicts that in this experiment firing fields should alternate across blocks so that units' response should be similar in the same the behavioral context (Fig. 5, top left). Conversely, temporal context theory predicts that in this experiment firing fields should change continuously without regard to the alternating behavioral contexts (Fig. 5, top right). For example, the first example cell (Fig. 5) had a firing field that was selective to samples of object 
2 when it was in the left position, and was most robust for the first two blocks of trials. That is, this unit fired across two blocks of trials with different behavioral contexts and then ceased firing despite the repetition of those behavioral contexts.

\section{Gradual changes in firing rate were uncorrelated with cluster stability or quality}

We tested the hypothesis that the above drift may signify signal instability that is due to either tetrode drift or poor unit isolation. First, we examined the relationship between cluster quality and spike rate drift by measuring isolation distance and $\mathrm{L}$ ratio and compared these metrics to drift in the average firing rate of each unit across $10 \mathrm{~s}$ bins time encompassing the entire recording. We failed to observe a relationship between either $\mathrm{L}$ ratio and spike rate drift (data not shown, Spearman's rho, $\left.r_{(768)}^{2}=-0.03, p>0.05\right)$ or isolation distance and spike rate drift across the population of pyramidal cells (Figures 6, 7, \& 8) (see Fig. $8 B ; r_{(768)}^{2}=$ $0.02, p>0.05)$. To exclude the possibility that unit activity drifted due to tetrode drift, spike clusters from each tetrode were carefully examined as a function of time. First, tetrodes that included waveform clusters that obviously appeared not stationary with regard to time were excluded (Manns et al., 2007). Then, drift in spike amplitude was correlated to drift in spike rate in the remaining units. For each cell, the Euclidean (4-dimensional) distance was measured between the average spike amplitudes (spike shape) of each $10 \mathrm{~s}$ bin in the session, and then the distances were regressed against their bin lag to obtain a measure of waveform drift (Fig. 6, right, red and blue line plots). To illustrate waveform drift, the first principal component of variance (PC) in the waveforms was calculated, and the average score of that first PC for waves in those same $10 \mathrm{~s}$ bins was plotted. The same was performed for the average firing rate at the same $10 \mathrm{~s}$ bins to obtain a measure of firing rate drift. To obtain a measure of spike-rate drift, the difference in firing rate between all pairs of bins was regressed against the lag between those bins. While there was a distribution of spike amplitude drift rates and activity drift rates in the population of pyramidal cells, there was no relationship between the two measures (see Fig. 7 \& Fig. 8A; Spearman's rho, $\left.r_{(768)}^{2}=0.0606, p>0.05\right)$.

These data revealed a wide spectrum of firing rate drift rates, as well as a wide
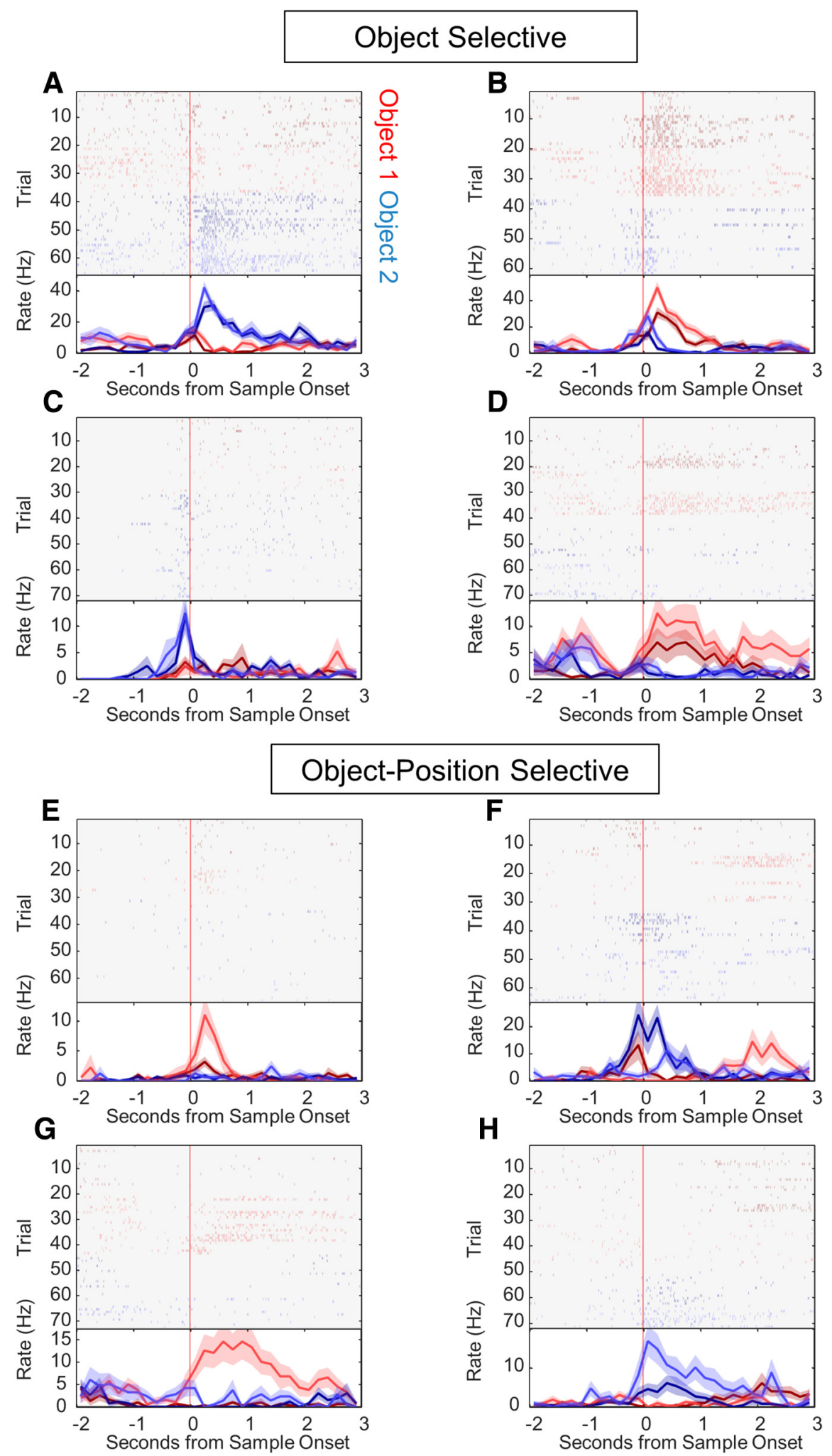

Figure 4. Many units showed object-specific firing $(\boldsymbol{A}-\boldsymbol{D})$ and conjunctive object-position firing $(\boldsymbol{E}-\boldsymbol{H})$. Perievent rasters and histogram plots centered on object sampling. Time refers to seconds from sample onset, and rate refers to firing rate in $\mathrm{Hz}$. We found some units to be object selective (red vs blue) regardless of the position of the object (light shades vs dark shades), as well as some objects to be selective to one object-position combination. Units $\boldsymbol{A}$ and $\boldsymbol{B}$ were recorded from Rat 4. Units $\boldsymbol{C}$ and $\boldsymbol{D}$ were recorded from Rat 1. Units $\boldsymbol{E}-\boldsymbol{G}$ were recorded from Rat 2. Unit $\boldsymbol{H}$ was recorded from Rat 3. 

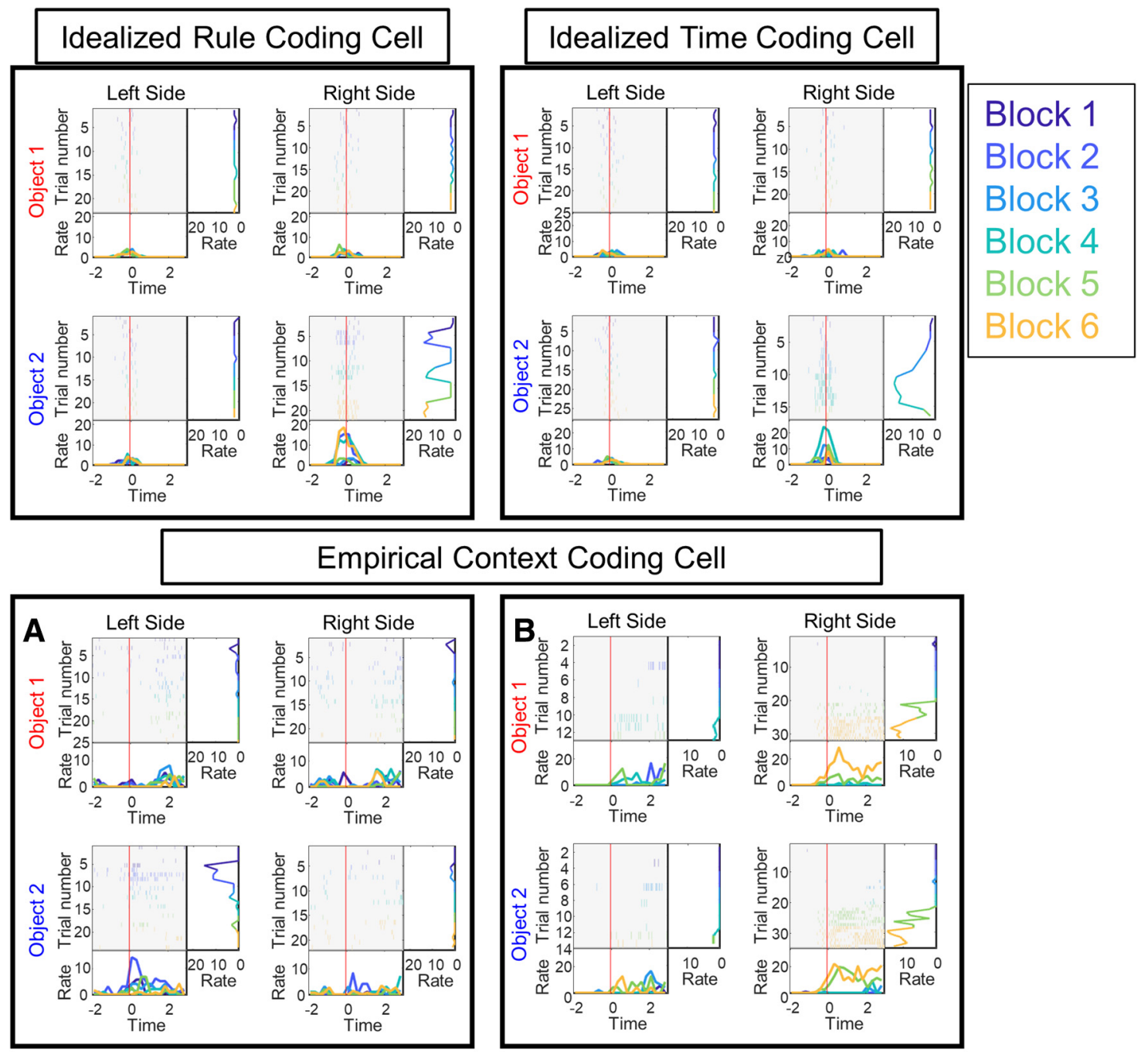

Context Coding Cell
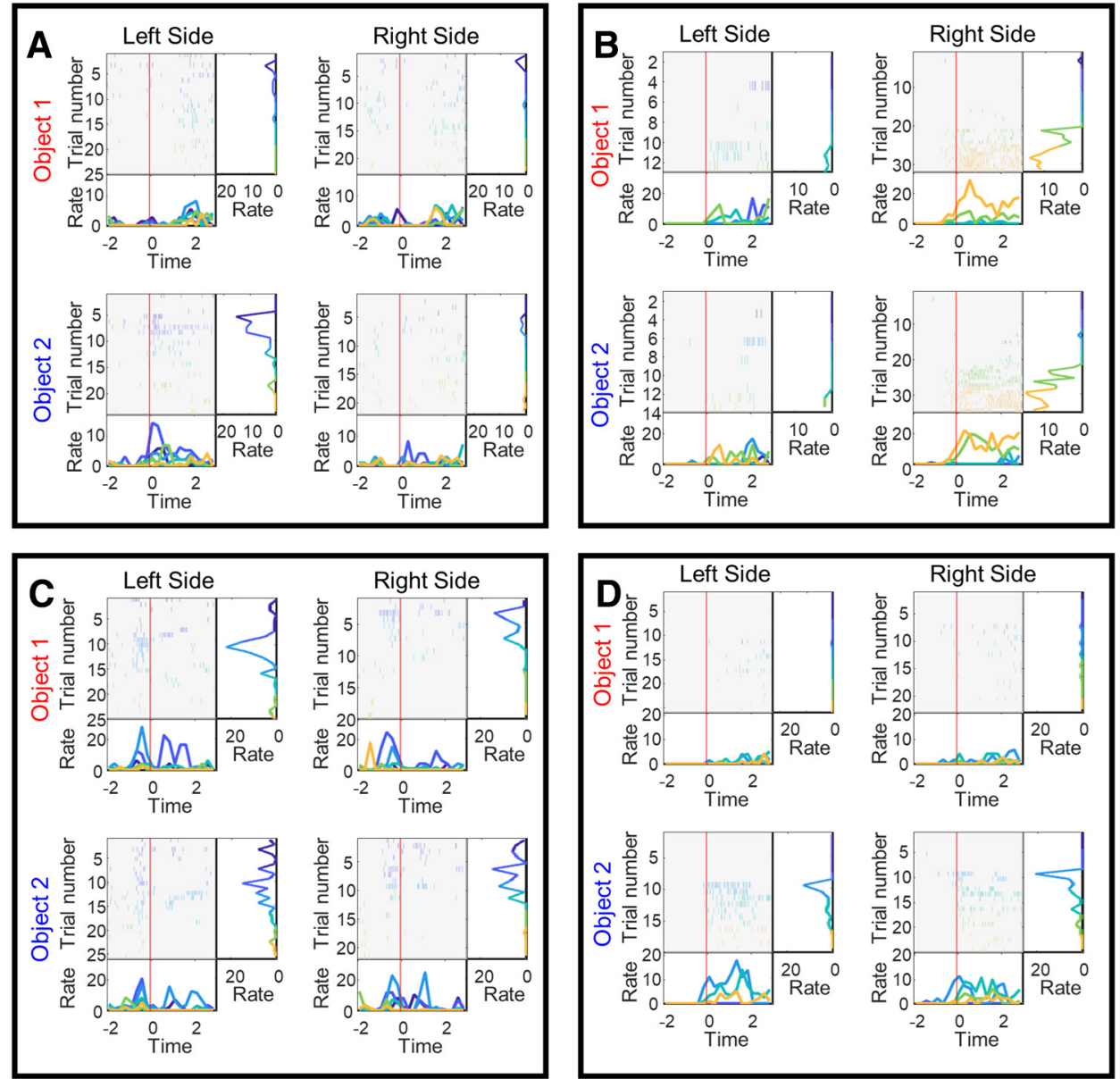

Figure 5. Many cells had context-dependent firing fields. Top, Idealized firing fields of dCA1 units in the temporally blocked object discrimination task. Each color in rasters and line plots represents samples in one block of trials (see Figs. 1A, 2). Top left, Ideal cell that responds selectively during one object-position combination, and does so only during blocks 2, 4, and 6 when object 2 is rewarded. Top right, Idealized object-position conjunctive cell that responds maximally during block 4 . Each idealized cell provides information about the object, position, and temporal structure of the task. $\boldsymbol{A}-\boldsymbol{D}$, Empirical cells showed firing fields that seemed to be centered on a small contiguous block of trials, suggesting temporal drift. No empirical cells showed firing fields that alternated with blocks, showing a lack of evidence for event segmentation. Units $\boldsymbol{A}, \boldsymbol{B}$, and $\boldsymbol{D}$ were from Rat 3. Unit $\boldsymbol{C}$ was from Rat 4 . All rates are in $\mathrm{Hz}$.

spectrum of spike amplitude drift rates. However, as there was no relationship between these two distributions, the single-unit data suggested that individual units exhibit a continuous spectrum of drift rates across time that cannot be explained by recording artifact.
Ensemble activity suggests hippocampal patterns slowly drift To supplement the analysis on individual units, population analyses were used to determine whether hippocampal ensembles showed evidence for event segmentation, a drifting temporal context, or both. Before performing these analyses, we removed 

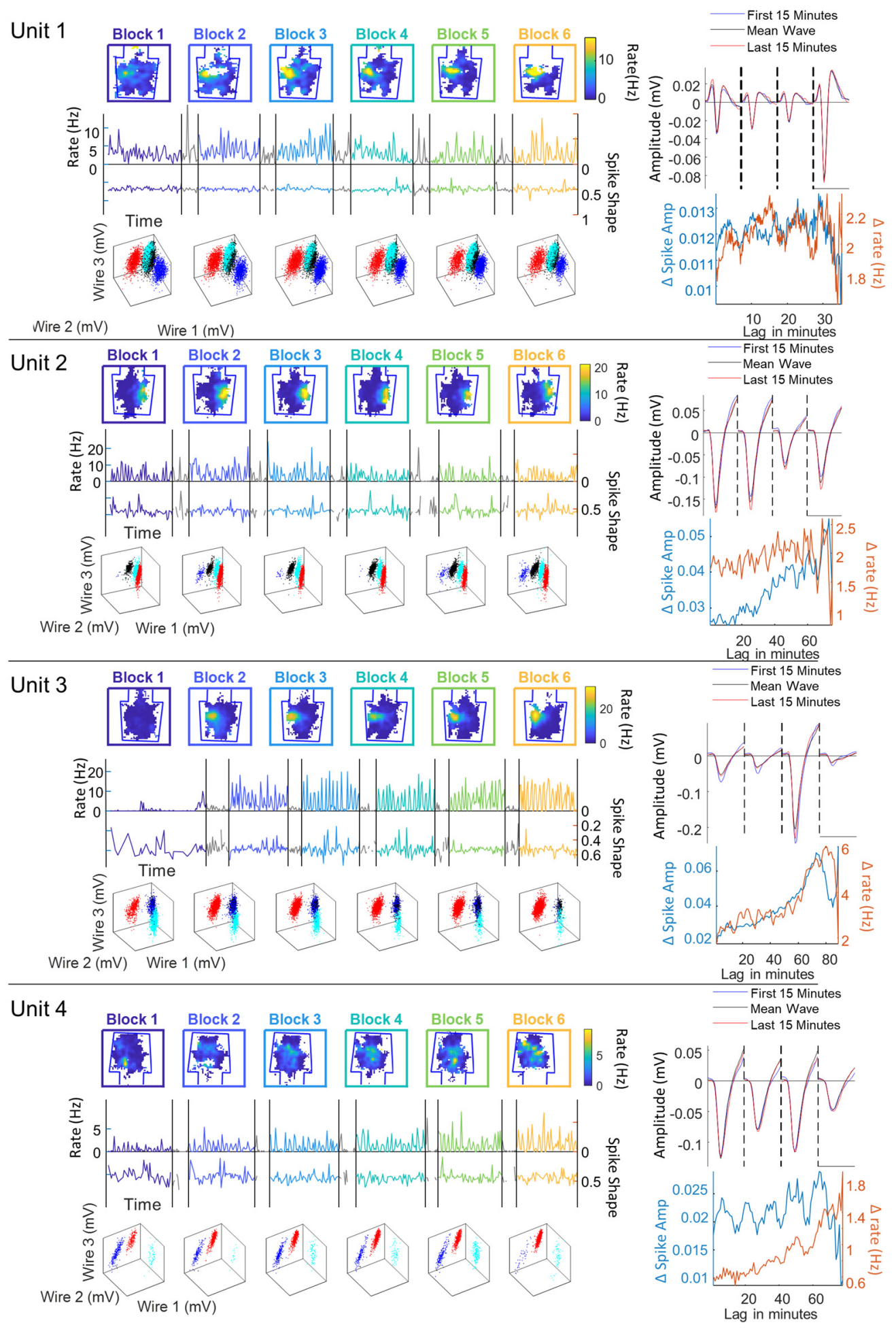

Figure 6. CA1 units exhibited a spectrum of drift rates both in firing rate and waveform shape. However, units that showed firing rate drift did not necessarily show waveform drift, and vice versa. Left, For each cell, the spatial heat map is shown for each block (top), the average spike rate and waveform shape for each $10 \mathrm{~s}$ bin across the recording session (middle), and spike amplitude clusters are shown for each block (bottom). Each example represents one cell at each extreme drift rate. Each unit cluster is represented in red (bottom for each unit), and all other units on that tetrode are marked in other colors. Top right, The average waveform shape on each of the 4 tetrode wires for each unit is illustrated. Calibration: Bottom right of waveforms, $800 \mu \mathrm{s}$. Bottom, The distance across bins for spike rate drift and waveform shape drift metrics was regressed against the increasing temporal lag between bins (see Materials and Methods). Cells showed a wide distribution of spike-rate drifts (red line on plot to the right) and spike shape drifts (blue line on plot to the right). However, the spike-rate drift was not related to spike shape drift. For instance, unit 4 shows a large amount of firing rate drift but stable waveform shape. In contrast, unit 2 shows a large amount of spike shape drift but does not show firing rate drift. All place plots (top for each unit) are normalized to a session maximum rate shown at right, and one pixel represents $3 \mathrm{~cm}$. Firing rate drift, waveform drift, and isolation statistics for each unit are as follows: unit 1 (from Rat 4 ): rate 0.03 , waveform 0.08 , L ratio $=10^{-4}$, isolation distance 118.62; unit 2 (from Rat 2): rate 0.02, waveform 0.91, L ratio $=10^{-3}$, isolation distance 31.53; unit 3 (from Rat 2): rate 0.90 , waveform 0.87, L ratio $=5 \times 10^{-3}$, isolation distance 32.76; unit 4 (from Rat 2): rate 0.93 , waveform 0.01 , L ratio $=2 \times 10^{-3}$, isolation distance 26.53 . 


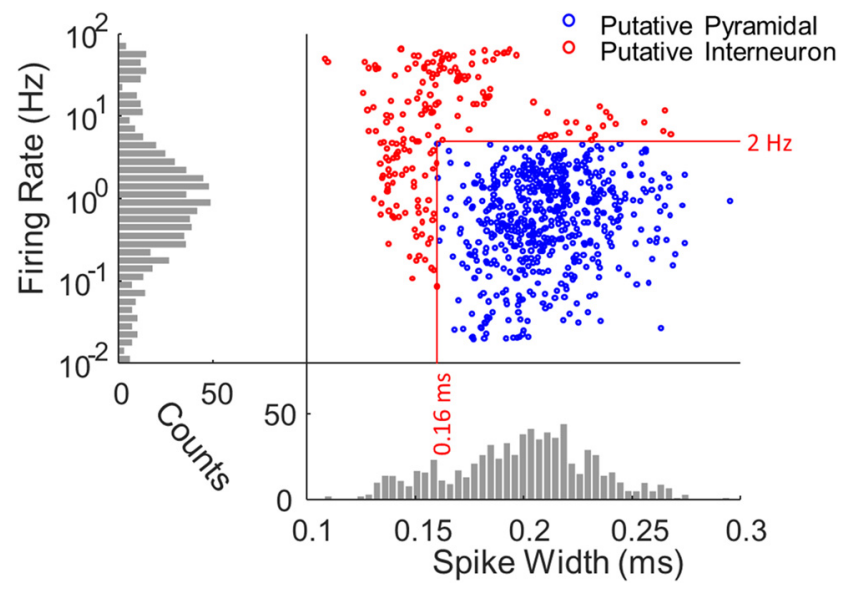

Figure 7. Pyramidal cells showed typical spike rates and waveform shapes. Interneurons were easily discriminated from pyramidal cells based on waveform width and firing rate. We adopted a $2 \mathrm{~Hz}$ and a 0.16 ms cutoff.

putative interneurons and kept only well-isolated units, leaving 531 putative pyramidal cells (see Materials and Methods). Event segmentation predicts that the hippocampus generates two stable representations, one for each rule condition (Fig. 9A, top left). This would manifest in alternating high correlations between blocks of the same rule condition, and low correlations between blocks of opposing rule condition. A drifting temporal context predicts that ensembles slowly change, and new representations would be continually generated (Fig. 9A, top right). This would manifest as a slow fall in the correlation between blocks at increasing temporal lag. We calculated the Spearman correlation of the population vector from each trial to all others to generate a correlation matrix for each rat (Fig. 9B; see Materials and Methods). We then averaged that matrix across all rats and sessions to generate a grand mean correlation matrix (Fig. 9A, bottom). The empirical pattern of ensemble activity showed strong support for drift, characteristic of temporal context (Fig. 9A, right) but no apparent evidence that representations from past blocks with the same reward contingency were repeated (Fig. 9A, left). Correlation values were greater at the beginning and the end of the session, but this may have been due to the lack of an acclimatization period at the start, and the fact that some sessions were shorter than others (Monaco et al., 2014). Importantly, the correlation values did not fall smoothly at increasing distances from the diagonal of the matrix. This was likely due to behavioral variables, such as where the rat was during the sampling event, and which pot the rat was sampling. Therefore, correlation matrices were also reorganized to control for these variables. First trials were sorted by position such that the first and fourth quadrants contained trial pairs in the same position. This revealed a strong ensemble code for space, as exemplified most strongly in Rat 3 and somewhat so in Rat 2 (Fig. 9B) where higher correlations were clustered at same position quadrants. The events were then sorted by object and last by time, to reveal correlations that fell more smoothly with increased distance from the diagonal (Fig. $9 B$, right). Furthermore, temporal proximity was evident across object and place representations in some rats as evidenced by yellow streaks parallel to the diagonal of the matrix, but away from the diagonal of the matrix (Fig. 9B, right; Rats 1, 2, 3, and less so in Rat 4). These stripes signify that temporally proximal trials that differed in object or place were represented more similarly than those that were farther apart in time. Thus, there was also appeared to be a portion of the ensemble that tracked trial lag across object or position.

\section{Hippocampal populations showed drift across blocks}

The effects observed in the correlation matrices were then quantified. If hippocampal ensembles reflected the two rule states, hippocampal activity would be more similar between blocks that shared a rule condition (at lags 2 and 4) versus those that involved opposing rule conditions (at lags 1, 3, and 5) (Fig. 10, top left). Conversely, if hippocampal ensembles tracked time, then hippocampal activity would become progressively less similar between blocks at increasing lag (Fig. 10, bottom left). The overall population correlation between blocks consistently fell as the block lag grew (Fig. 10, right, purple line) (bootstrap permutation test, slope $=-0.031$, observed slope exceeded all 10,000 permutations, permutation mean $\left.1.71 \times 10^{-5}, \sigma=0.0043\right)$. The representation for the same behavior (e.g., same object, position, and response) also progressively decorrelated with block (Fig. 10, right, yellow line) (slope $=-0.049$, the observed slope exceeded all 10,000 bootstrapped permutation permutation mean $=1.9 \times$ $\left.10^{-5}, \sigma=0.0064\right)$. When these results were reevaluated after only using units with the most stable waveform clusters (most stable quartile after controlling for object and position, see Fig. $8 A$, yellow line), the population still significantly decorrelated with increasing temporal lag, and at a similar rate (slope $=$ -0.030 , observed slope exceeded all 10,000 perms, permutation mean $\left.6.62 \times 10^{-5}, \sigma=0.0067\right)$. Similarly, the representation of each delay in between blocks also progressively decorrelated with increasing lag (Fig. 10, black line; slope of correlation vs lag = -0.039 , observed slope exceeded all 10,000 perms, permutation mean $\left.=-1.4 \times 10^{-5}, \sigma=0.00065\right)$. Previous studies have observed changes in the hippocampal spatial map in the first trials of a recording session (Mehta et al., 2000; Monaco et al., 2014). To account for this potential confound, we also examined correlations after removing the first block of trials on each session. Indeed, after controlling for object, position, and response (e.g., Fig. 10, yellow curve), the population still significantly decorrelated with increasing temporal lag (slope $=-0.038$, observed slope exceeded all 10,000 perms, permutation mean $=2.0 \times$ $\left.10^{-5}, \sigma=0.0071\right)$. It is also possible that small but significant changes in the spatial occupancy of the rat across time were responsible for the changes in cell activity across blocks. Considering a minority of sessions (7, see previous section) included slow changes in the rat's start position across trials, we also performed these analyses after removing those sessions. Neural ensembles from the remaining sessions also significantly decorrelated with time (same object, and position correlation slope vs lag $=$ -0.045 , observed slope exceeded all 10,000 perms, permutation mean $\left.=1.0 \times 10^{-4}, \sigma=0.0077\right)$. Correlations for the same item, position, and response remained higher than those across all sample types at each block lag (Bonferroni-corrected ranksum test: lag 1: within object, position, response mean correlation $=0.37$, across mean correlation $=0.22, p<5 \times 10^{-4}$, lag 2 within mean $=0.31$, across mean $0.16, p<5 \times 10^{-4}$, lag 3 within mean $=0.24$, across mean $=0.11, p<5 \times 10^{-4}$, lag 4 within mean $=0.19$, across mean $=0.06, p<5 \times 10^{-4}$, lag 5 within mean 0.14 , across mean $=0.05, p<0.005)$, suggesting a robust code for objects and places, even though the overall population drifted. Therefore, all empirical curves replicate previous findings of a temporal context coded in conjunction with a stable code for items, places, and behavior. Deviations from the smoothly decreasing curves are small; any contribution from discrete event coding would have to be much smaller than the con- 

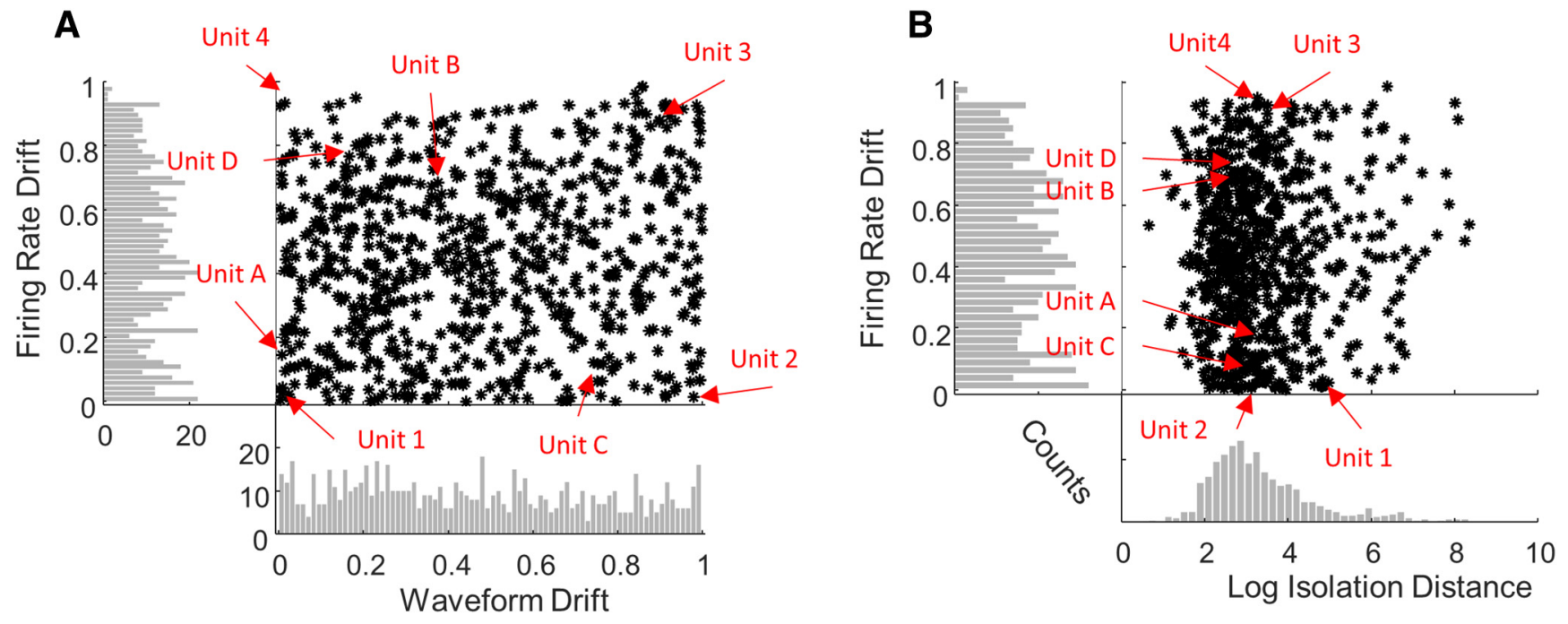

Figure 8. Firing rate drift was unrelated to waveform drift or isolation distance. $\boldsymbol{A}$, Firing rate drift was uncorrelated to waveform drift. Firing rate drift was calculated as the correlation between the absolute difference in firing rate between 10 s bins and the difference in time between those bins. Waveform drift was calculated as the correlation between the absolute Euclidean distance between the waveform shape between 10 s bins and the difference in time between those bins. $\boldsymbol{B}$, Firing rate drift was also uncorrelated to isolation distance. For firing rate drift, waveform drift, and isolation distance calculations, see Materials and Methods. Unit references in $\boldsymbol{A}$ refer to those units in Figure 5 (unit A:D) and Figure 6 (unit 1:4).

tribution due to gradually changing temporal context. Moreover, event segmentation would predict that the deviations from a smooth curve should be consistent from one type of comparison with the other, resulting in parallel curves (Fig. 10, top left). However, to the extent there were deviations in the different empirical curves, they were not systematic across the type of comparison. Thus, consistent with behavior, there was no evidence that hippocampal populations represented blocks in discrete segments based on the two rule conditions.

Overall hippocampal ensembles showed strong temporal drift with no evidence that past blocks of the same rule condition used the same code. This was true for the overall population but was also found in conjunction with a code for places, objects, and object-place conjunctions. Furthermore, the code for objects and positions remained across all blocks. Thus, even though hippocampal populations drifted from block to block, there remained a code for objects and positions that persisted throughout the recording. Temporal drift also persisted during the interblock delays where there was also no evidence for an alternating neural structure.

\section{Hippocampal populations showed drift within block}

The foregoing analyses demonstrate that the hippocampal ensemble changed gradually across blocks on the scale of tens of minutes. This subsection examines changes in hippocampal representation within a block on the scale of $\sim 1$ minute. To replicate previous findings, population correlations were compared between individual trials (Fig. 11) (Manns et al., 2007). There was significant temporal drift in the overall representations across trials (Fig. 11, purple line; observed slope $=-0.0091$, observed data exceeded all 10,000 perms, permutation mean $=1.5 \times$ $\left.10^{-5}, \sigma=9.5 \times 10^{-4}\right)$. Temporal drift within a block was apparent after controlling for object (Fig. 11, blue line; observed slope 0.009 , observed data exceeded all 10,000 perms, permutation mean $=-8.46 \times 10^{-6}, \sigma=1.3 \times 10^{-3}$ ), position (Fig. 11, green line; observed slope $=-0.011$, observed data exceeded all 10,000 perms, permutation mean $=2.18 \times 10^{-5}, \sigma=1.2 \times 10^{-3}$ ), and object, and position (Fig. 11, yellow line; observed slope $=$ -0.015 , observed data exceeded all 10,000 perms, permutation mean $\left.=8.1 \times 10^{-6}, \sigma=1.5 \times 10^{-3}\right)$. Drift was also apparent across exclusively correct trials (data not shown; observed slope $=$ -0.012 , observed data exceeded all 10,000 perms, mean $=3.3 \times$ $10^{-5}, \sigma=2.3 \times 10^{-3}$ ), suggesting that this is not an artifact of the learning curve observed in Figure 2. Furthermore, this minute-to-minute drift was even apparent in the most stable clustered units and after controlling for object and position (only units that were in the top quartile of waveform stability, see Fig. $8 A$ ) (observed slope $=-0.0058$, observed data exceeded all 10,000 perms, permutation mean $=-9.3 \times 10^{-6}, \sigma=6.8 \times$ $\left.10^{-4}\right)$. Considering there was a small minority of blocks in which the rat's start position changed systematically, it is possible that this change in behavior may have contributed to the neural drift. However, gradual drift remained prominent, even after removing all blocks in which the rat's starting position changed across time and controlling for object and position effects (after removing the 6 unstable blocks of 150, observed slope $=-0.0146$, observed data exceeded all 10,000 perms, permutation mean $=$ $\left.-5.1 \times 10^{-5}, \sigma=0.0025\right)$ This drift occurred individually in the overall neural population of each rat (observed slopes $=-0.012$, $-0.011,-0.0074,-0.0075$, all observed data exceeded all 10,000 perms).

These data replicate previous findings that show drift is observable across individual trials. Drift on the order of seconds was observed in the overall code, as well as the code for objects, positions, and object-position conjunctions. Thus, reliable population drift was observed, even across iterations of the same behavior and context, and over a scale of seconds.

\section{Hippocampal shifts between blocks can be accounted for by time}

We observed robust drift in the hippocampal population occurring both across blocks of trials as well as on a trial-to-trial basis. However, it is possible that, in conjunction with drift within each block, there exist shifts in population state at the transitions between blocks (see Fig. 1, Segmented Temporal Context vs Drifting Temporal Context). This would manifest as an increase in the population drift rate across blocks over what was observed within a block. To isolate this possible effect, trial pairs within a block 
A
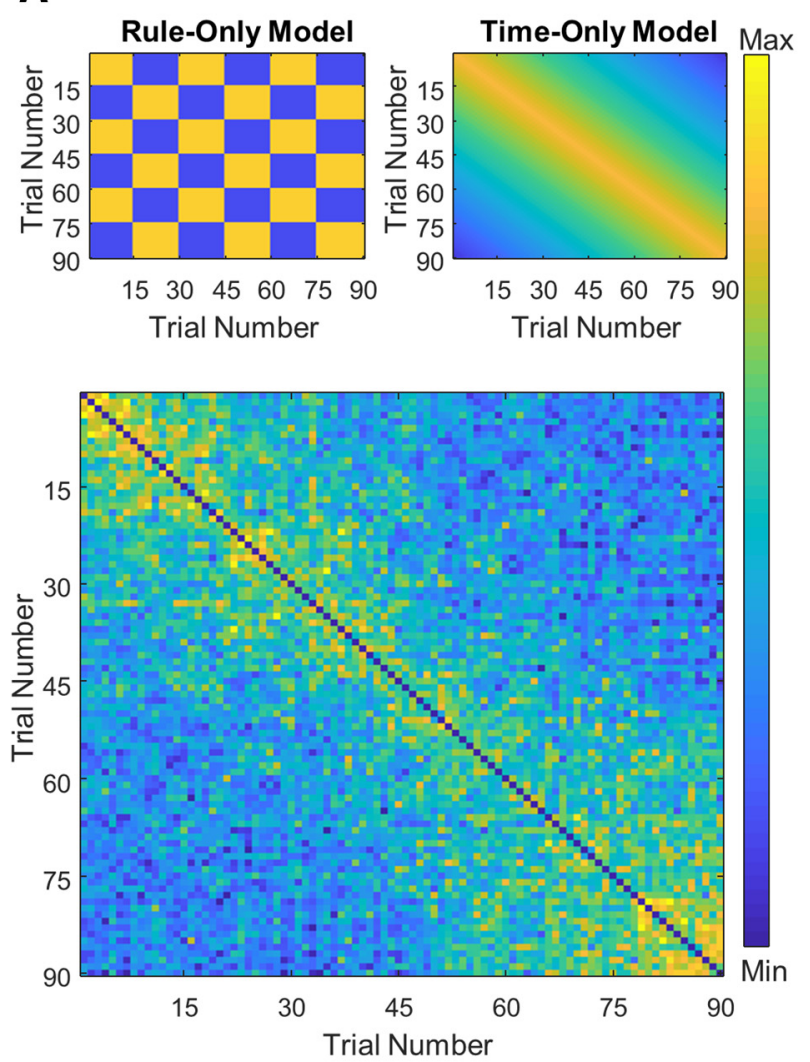

B

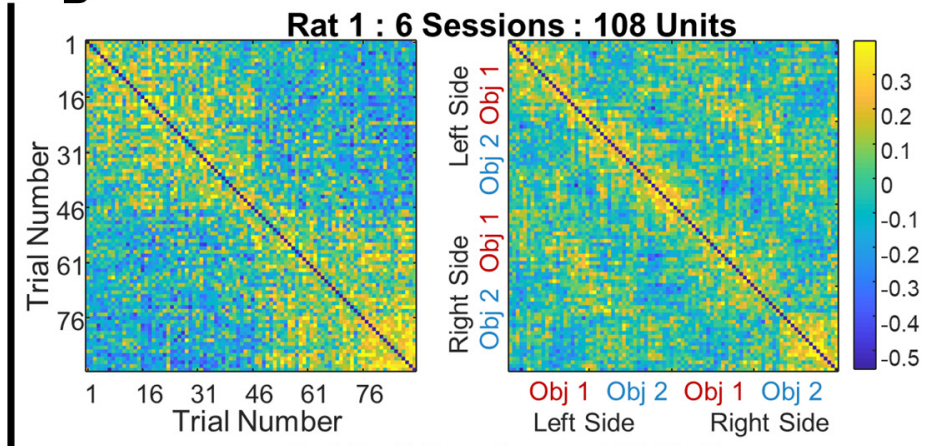

Rat $2: 8$ Sessions : 165 Units
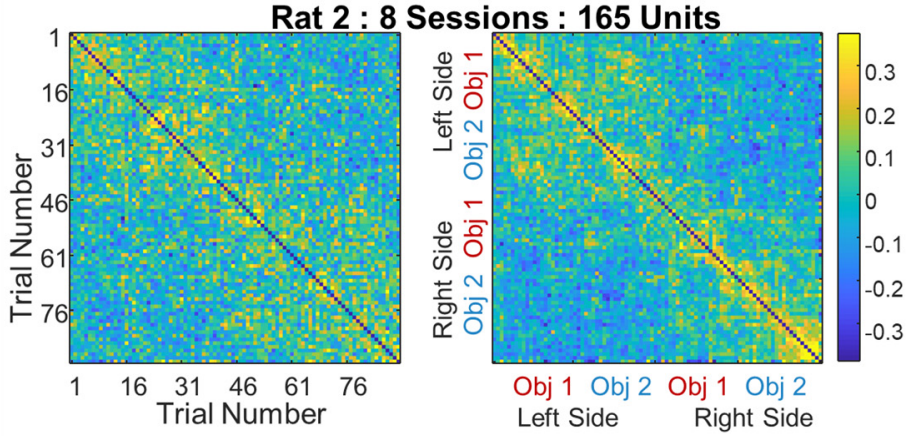

Rat $3: 7$ Sessions : 223 Units
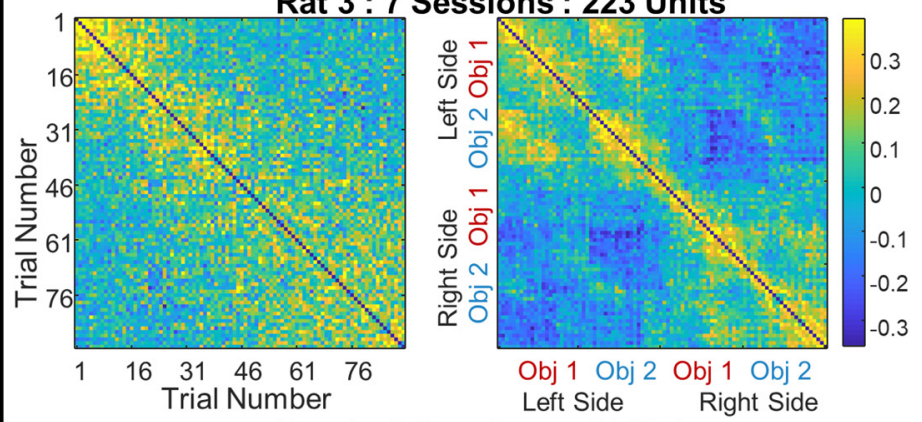

Rat 4 : 2 Sessions : 29 Units

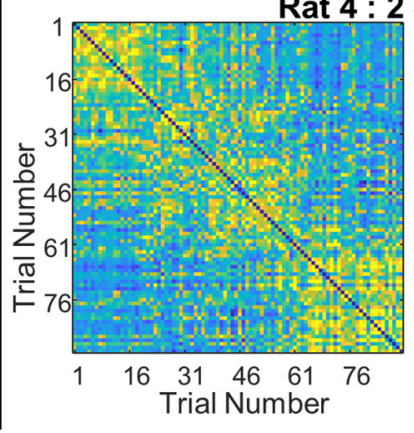

Figure 9. Drift, and not a representation of the repeating rule conditions, was apparent in correlation matrices. $A$, Observed correlation matrix suggests continuous temporal drift. Top left, Predicted correlation matrix if ensembles represent a context code consistent with event segmentation. Top right, Predicted correlation matrix if ensemble code is consistent with drifting temporal context. Bottom, Empirical correlation matrix averaged across animals more closely resembles predicted matrix under the temporal context hypothesis. $\boldsymbol{B}$, Trial-by-trial correlation matrices for each rat also show temporal drift. Left, Matrices were sorted by trial number as was done in $\boldsymbol{A}$. Right, Matrices sorted by position, then by object, then by trial number. Position coding can be seen as higher correlations in the top left and bottom right quadrants of each matrix. Object coding can be seen as nested quadrants within each position quadrant. Once trials were sorted by object and place, high correlations still clustered adjacent to the eye of the matrix, indicating that ensembles were more similar between trials at closer temporal proximity. Similarity was calculated by generating $z$-normalized firing rate vectors for each cell across sampling events, and then calculating the Spearman's rho between the population vectors on each trial. The color scale is equivalent across the two matrices for each rat, but a different scale was used for each rat.

(Fig. 12, left, blue line) were examined separately from those that spanned a block transition (Fig. 12, left, red line). We observed significant drift both within each block as well as across blocks ( ithin block observed slope $=-0.009$, observed data exceeded all 10,000 perms, permutation mean $=-1.7 \times 10^{-6}, \sigma=3.5 \times$ $10^{-4}$; across block observed slope $=-0.0032$, observed data exceeded all 10,000 perms, perm $=-1.66 \times 10^{-6}, \sigma=2.1 \times$ $10^{-4}$ ). After controlling for trial lag, the block transitions induced a separation in representations of contexts as other studies have suggested (Baldassano et al., 2017). Because quantification of segmentation is inversely related to that of drift in this instance, choosing segmentation as the alternative hypothesis leaves drift 

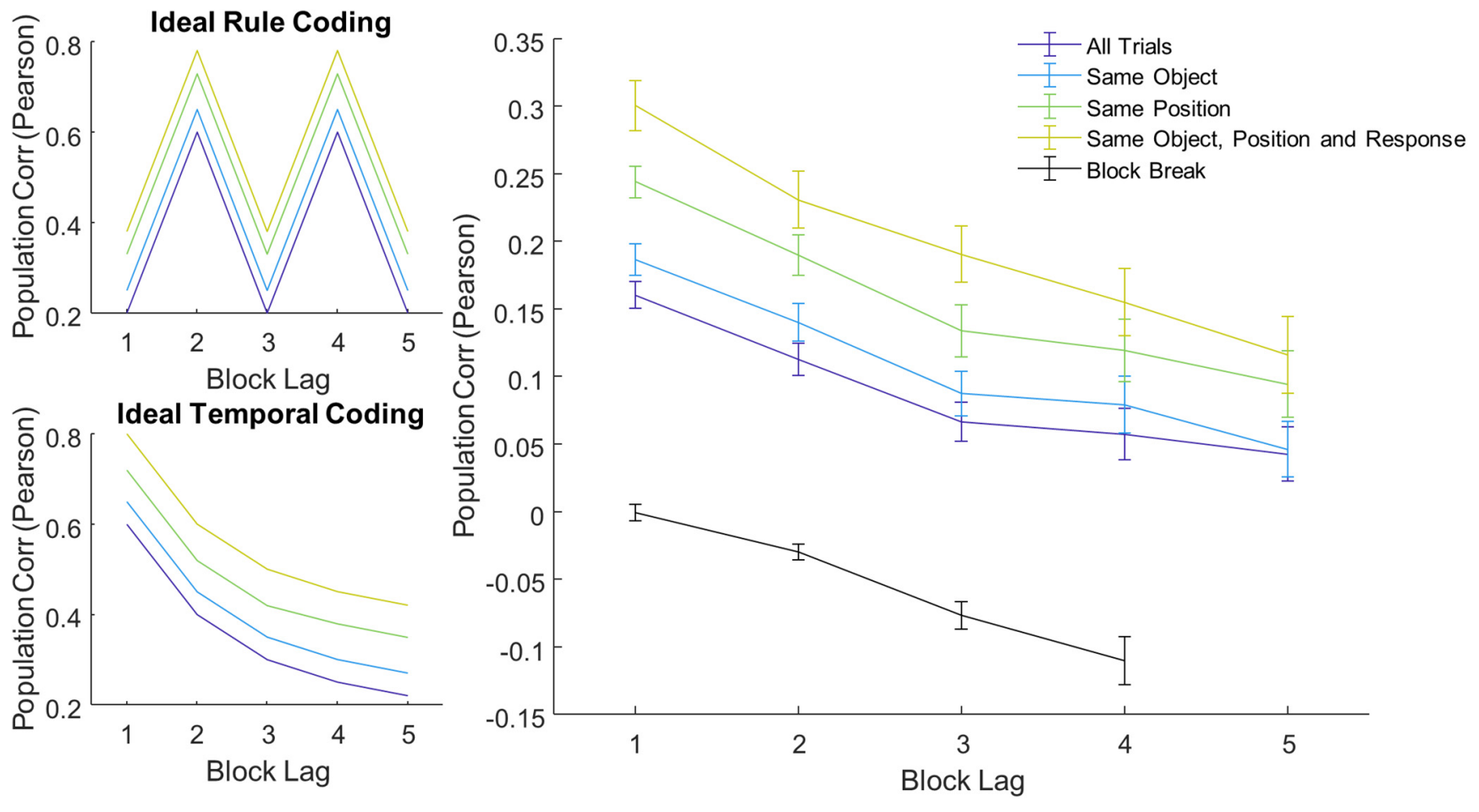

Figure 10. Ensembles reflected context code that continually changed across blocks and did not recur. Top left, Ideal curves under event segmentation hypothesis. Event segmentation predicts high correlations between blocks of the same rule condition at lags 2 and 4 . Bottom left, Ideal curves under the contextual drift hypothesis. Contextual drift predicts a monotonic decrease in each curve. Right, Observed curves for ensemble correlations fell as the block lag between the trials grew. This was true for the overall population (purple line) as well as in the ensemble coding the same object (blue) or position (green) or same object, position, and behavioral response (yellow), and finally the intervals between the blocks (black line). All curves represent a systematic decrease from small to large lags with no obvious alternation. To the extent that there are fluctuations in the curves, these fluctuations were small and not consistent across different types of comparisons. Error bars represent SEM.

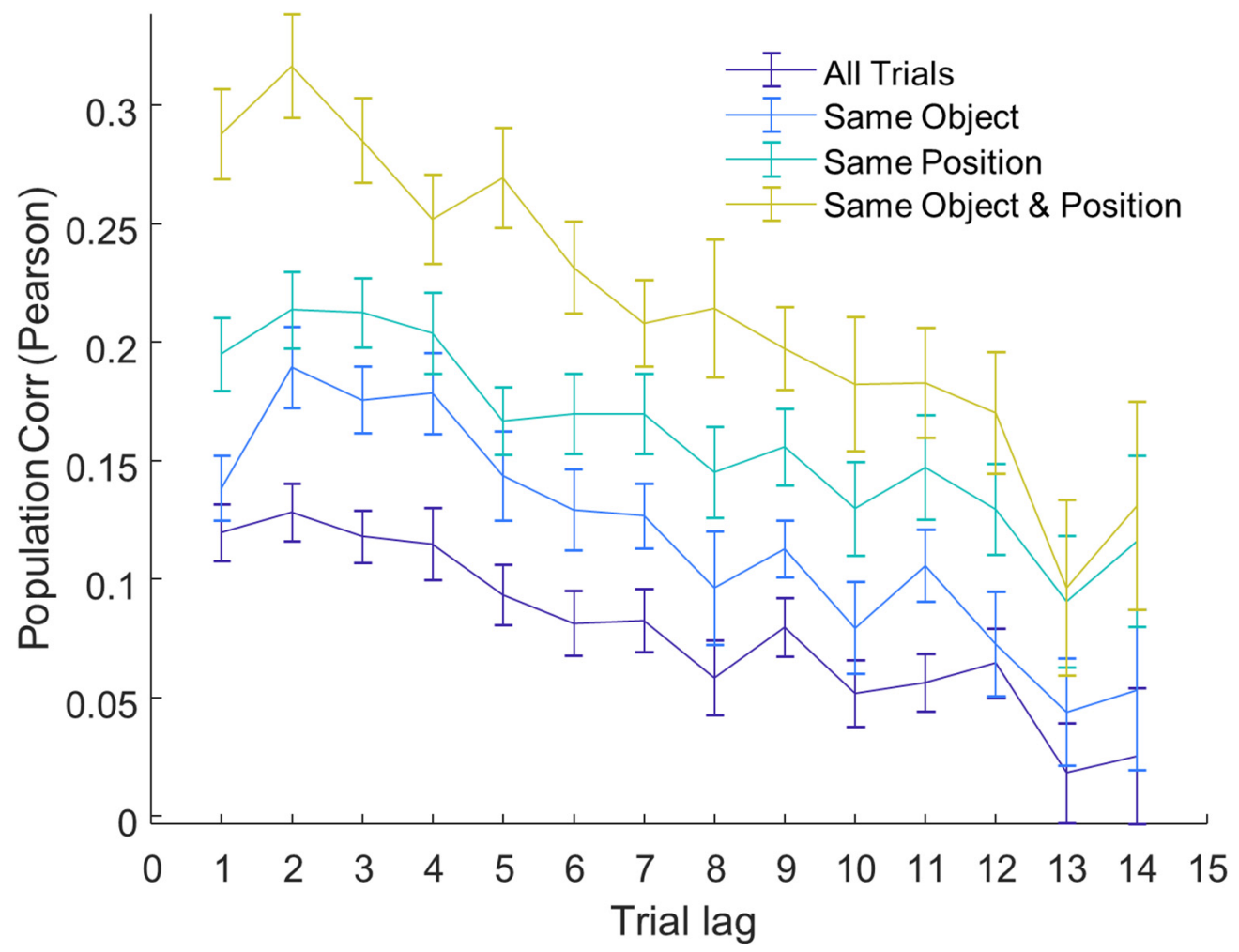

Figure 11. Ensemble correlations within a block reliably fell as trial lag increased. This effect was evident both in the overall population activity (purple line), as well as when we controlled for object (blue), position (green), or object, position, and response (yellow). Error bars indicate SEM. 

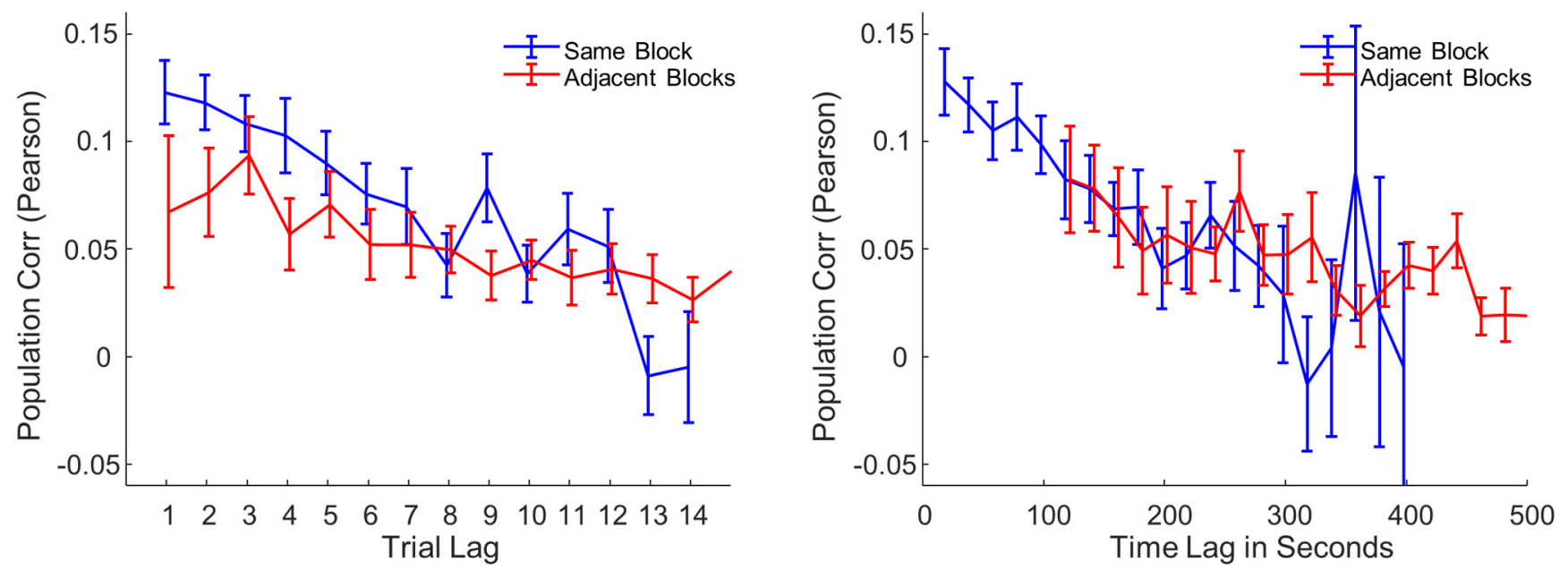

Figure 12. Time fully accounts for shifts in population state across blocks. Left, Population vectors within a block (blue) were more similar than those in adjacent blocks (red) when trial lag was considered. Alone, this might have been evidence for event segmentation. Right, Population vectors within block (blue) were not more similar than those in adjacent blocks (red) when time was considered. Population vector correlations were lower in adjacent blocks, but time was sufficient to account for this decrease. Thus, there was no evidence for event segmentation between blocks above and beyond the change attributable to the temporal delay between blocks. Population vectors were constructed in the same manner as in Figure 11 (purple line). Error bars indicate SEM.

as the null. Standard statistical testing was therefore problematic, as it provides only positive evidence for one alternative hypotheses over the null. Therefore, we used a Bayes factor to directly compare the likelihoods of segmentation and drift and find positive evidence for the more likely hypothesis, equally assessing the alternative and null. The correlation between trials in the same block was significantly greater than that in adjacent blocks after controlling for trial lag (JZS Bayes T test yielded a Bayes factor weakly in favor of a difference in means, odds ratio 3.2:1). This suggests that the transition between blocks may have induced a separation between representations of trials that happened in different blocks consistent with event segmentation.

This segmenting effect could either be because the boundary cue induced a separation in hippocampal representations or that the separation in representations was merely a consequence of the extended time between the two trials spanning the delay. To address whether the extended temporal lag fully accounted for the apparent boundary effect on the hippocampus, we plotted the correlation between trials by their temporal lag and then organized pairs by whether there was a block transition between them (Fig. 12, right). When we compared trial pairs in the same block with those in adjacent blocks in this way, we found that the block break caused no more reduction in the population vector correlation than would be expected by elapsed time (JZS Bayes T Test yielded a Bayes factor strongly in favor of a single mean, odds ratio 10.8:1). Thus, the separation of trials spanning a block transition observed in the left figure was completely eliminated by accounting for elapsed time. This was also true after removing trials at the beginning of the block when performance was poor (trials 4:15 of each block only, JZS Bayes T test yielded a Bayes factor strongly in favor of a single mean, odds ratio 6.54:1) removing the possibility that the representation of context only shifted after the animal had switched behavioral strategy. Thus, even though every animal reliably alternated their choices between blocks and no rat perseverated into the next block, there was no evidence that hippocampal code segmented experience any more than what would be expected from elapsed time.

\section{Discussion}

This experiment sought to examine the hippocampal code for behaviorally relevant context in a task where animals were re- quired to segment experience to distinguish between two rule contingencies. Event segmentation predicts animals would parcellate experience into discrete episodes based on contextual boundaries; temporal context predicts that the hippocampal representation should change continuously across time.

Well-trained animals neither perseverated across cued block transitions nor anticipated the rule reversal. Instead, all rats began every block at chance, frequently digging in the first pot encountered. Thus, while no rat learned there were two alternating rule conditions, they all benefitted from the boundary cue, changing their behavior across blocks. Hippocampal units showed selectivity for the position, object, and object-position conjunction of sampling events while also showing sensitivity to the changing context. The ensemble code showed strong evidence for temporal drift both across blocks and within blocks. There was no evidence that the ensemble code was more similar for blocks that shared a rule contingency. Furthermore, there was no greater separation in the hippocampal representation than was expected by the passage of time across block transitions.

\section{The methodological design could have prevented a segmented neural representation}

The behavioral results suggest that, while rats did not learn to anticipate the reversal of the reward contingency between blocks, they were sensitive to the boundaries between blocks. There are multiple interpretations of these results. One clue was that rats often chose the first pot encountered at the start of each block, suggesting that rats forgot the past rule condition during the block transition. Alternatively, they may have grown impatient during the delay, and were unwilling to reject either pot. Previous data suggest that this behavior may reflect a hippocampaldependent cognitive flexibility. Numerous studies have shown that hippocampal lesions cause rats to perseverate more after switches in rule contingency or reward location (Kimble and Kimble, 1965; Hsiao and Isaacson, 1971). Considering our animals showed no consistent perseveration across blocks, these results suggest that the dorsal hippocampus might have supported the savings the rats exhibited at the beginning of each block.

Further support for this interpretation may be found in studies on the recency effect that also show a hippocampal-dependent memory strengthening for recent list items (Kesner et al., 1988). 
Under this interpretation, the delay weakened the rat's memory and contributed to the rat's uncertainty at the beginning of each block. These two accounts suggest that, even though animals could not track the two recurring rule conditions, their behavior may have been supported by hippocampal processing.

\section{This experiment differed from other context-dependent experiments}

Consistent with behavior, we found no support in the activity of hippocampal ensembles for a rule-specific code. These data are in contrast to previous experiments that presented animals with distinct behavioral contexts (e.g., Markus et al., 1995; McKenzie et al., 2014). However, it is important to note the difference between the contextual cues in this experiment and those in previous experiments. Those experiments that did observe segmentation used spatially distinguishable contexts (Komorowski et al., 2009, 2013; McKenzie et al., 2014) or overt external cues to discriminate the context, such as an ambient sound, odor, or object (McNaughton et al., 1996; Zaremba et al., 2017). The present task was specifically designed to be devoid of such ambient cues, as cue responses could be mistaken for a new context signal providing false evidence for event segmentation. Other tasks generated contexts consisting of separate behavioral tasks or event sequences in the same environment (Markus et al., 1995; Ferbinteanu and Shapiro, 2003; Bower et al., 2005; Dupret et al., 2010). In those paradigms, context was designated by changes in the physical behavioral sequences and reward locations (Markus et al., 1995; Kobayashi et al., 1997). Thus, trajectory-dependent firing "splitting" may have been responsible for the context signal in these experiments (Ferbinteanu and Shapiro, 2003; Grieves et al., 2016). As the spatial layout of this task was held constant across blocks, there were no systematic changes in the animal's trajectory, which could explain the absence of an alternating neural code.

A previous study found that, in rats performing alternation behaviors, alternation in the hippocampal code was not always apparent and was sensitive the location of the reward itself (Bower et al., 2005). That study suggests that the shaping procedure in rodent experiments has a profound effect on context disambiguation in the hippocampus (Bower et al., 2005). Perhaps rats benefit from the opportunity to first explicitly learn two alternating rule conditions through training with an explicit contextual cue, such as wall color, or ambient sound. Rats might then continue to track the alternating context, even after the ambient contextual cues are removed. This might also reveal a hippocampal representation of the two contexts and provide evidence for event segmentation when only boundary cues segment context. On the other hand, the hippocampus may code temporal context in a unique manner, and other regions, such as the lateral entorhinal cortex, may have shown segmentation in this task (Tsao et al., 2018).

\section{These data add to a growing temporal drift literature}

Hippocampal populations showed robust drift through time both across seconds as well as minutes. These data add to reports that suggest that hippocampal representations change across time. Importantly, temporal drift has been observed in experiments using a variety of recording methodologies (Manns et al., 2007; Ziv et al., 2013; Mankin et al., 2015; Rubin et al., 2015; Cai et al., 2016; Mau et al., 2018). Hippocampal drift has also been observed in both hippocampal-dependent tasks as well as tasks without mnemonic demands (Manns et al., 2007; Mankin et al., 2012; see also Tsao et al., 2018). Figure 6 showed the first direct evidence that population drift is uncorrelated with waveform drift in a chronic tetrode recording preparation. Further suggesting that these time signals are unlikely to be a recording artifact, many units observed in the lateral entorhinal cortex by Tsao et al. (2018) showed repeated slow changes in spike rate that were triggered by entry into a new environment. These studies raise the possibility that population drift reflects a functional correlate of hippocampal processing that always occurs.

A drifting representation of context supports the behavioral results in this experiment, suggesting that this hippocampal process may have contributed to the observed behavioral flexibility. This representation was supported on the individual unit level via slow changes in conjunctively selective object-position specific firing fields. Drift occurred both in these units coding for both the position and identity of objects as well as in units without obvious place or object selectivity. Crucially, some firing fields remained stable; and across the population, there was a spectrum of drift rates (Fig. 6). On the one hand, there remained a stable population that coded for objects and positions across the session. On the other hand, a drifting ensemble provided a continuous dimension for relating experiences and is a likely mechanism for tracking the temporal relationships of events across many scales of time (Cai et al., 2016; Eichenbaum, 2017). Drift enables rats to associate recent trials occurring in the same block and disassociate distant trials from previous block.

\section{Was the hippocampus really insensitive to the event segmenting cues?}

If the animals generated new contextual representations at the onset of each block of trials, one might hypothesize that the boundary cue would impact the hippocampal code. Recent modeling suggests that event segmentation may occur when the actor detects shifts in the latent causes defining a context, and that the hippocampus is necessary to properly assign a new context (Gershman and Niv, 2010; Gershman et al., 2017). Indeed, the boundary cue, in this case, the prolonged delay and removal from the task environment, did impact the rats' expectations, as no rat showed response perseveration on the first trial of a block. fMRI studies in humans suggest that this effect is caused by increased hippocampal activation coinciding with recognition of an event boundary (Swallow et al., 2011; Baldassano et al., 2017). This boundary effect has been recently replicated in the rodent hippocampus during two paradigms where space served to contextualize experience (Place et al., 2016; Bulkin et al., 2018). In this experiment, there was an increase in drift between trials separated by a block boundary, but the increased separation in representations was completely accounted for by the passage of time between blocks. This result suggests that removal from the environment had no impact on the hippocampal representation. However, an alternative explanation is that time was sufficient to separate the hippocampal representation of context and promote a change in the rats' expectations. Perhaps the temporal delay between blocks somehow inhibited the identification of a discrete change in the latent cause governing the reward contingency. Under this interpretation, other cues may separate contexts if they were only given the right opportunity. Future experiments could dissociate the impact of each of these cues by imposing either the prolonged delay or the removal from the environment alone, or both in combination to observe different levels of separation in the hippocampal representation across time. 


\section{References}

Baldassano C, Chen J, Zadbood A, Pillow JW, Hasson U, Norman KA (2017) Discovering event structure in continuous narrative perception and memory. Neuron 95:709-721.e5.

Bower MR, Euston DR, McNaughton BL (2005) Sequential-contextdependent hippocampal activity is not necessary to learn sequences with repeated elements. J Neurosci 25:1313-1323.

Brandon MP, Koenig J, Leutgeb JK, Leutgeb S (2014) New and distinct hippocampal place codes are generated in a new environment during septal inactivation. Neuron 82:789-796.

Bulkin DA, Sinclair DG, Law LM, Smith DM (2018) Hippocampal state transitions at the boundaries between trial epochs. BioRxiv 1:11.

Cai DJ, Aharoni D, Shuman T, Shobe J, Biane J, Song W, Wei B, Veshkini M, La-Vu M, Lou J, Flores SE, Kim I, Sano Y, Zhou M, Baumgaertel K, Lavi A, Kamata M, Tuszynski M, Mayford M, Golshani P, Silva AJ (2016) A shared neural ensemble links distinct contextual memories encoded close in time. Nature 534:115-118.

Davachi L (2006) Item, context and relational episodic encoding in humans. Curr Opin Neurobiol 16:693-700.

Deuker L, Bellmund JL, Navarro Schröder T, Doeller CF (2016) An event map of memory space in the hippocampus. Elife 5:1-26.

DuBrow S, Rouhani N, Niv Y, Norman KA (2017) Does mental context drift or shift? Curr Opin Behav Sci 17:141-146.

Dupret D, O’Neill J, Pleydell-Bouverie B, Csicsvari J (2010) The reorganization and reactivation of hippocampal maps predict spatial memory performance. Nat Neurosci 13:995-1002.

Eichenbaum H (2017) On the integration of space, time, and memory. Neuron 95:1007-1018.

Eichenbaum H, Yonelinas AP, Ranganath C (2007) The medial temporal lobe and recognition memory. Annu Rev Neurosci 30:123-152.

Ezzyat Y, Davachi L (2011) What constitutes an episode in episodic memory? Psychol Sci 22:243-252.

Ferbinteanu J, Shapiro ML (2003) Prospective and retrospective memory coding in the hippocampus. Neuron 40:1227-1239.

Folkerts S, Rutishauser U, Howard MW (2018) Human episodic memory retrieval is accompanied by a neural contiguity effect. J Neurosci 38 : $4200-4211$.

Gershman SJ, Niv Y (2010) Learning latent structure: carving nature at its joints. Curr Opin Neurobiol 20:251-256.

Gershman SJ, Monfils MH, Norman KA, Niv Y (2017) The computational nature of memory modification. Elife 6:e23763.

Grieves RM, Wood ER, Dudchenko PA (2016) Place cells on a maze encode routes rather than destinations. Elife 5:e15986.

Howard MW, Fotedar MS, Datey AV, Hasselmo ME (2005) The temporal context model in spatial navigation and relational learning: toward a common explanation of medial temporal lobe function across domains. Psychol Rev 112:75-116.

Howard MW, Youker TE, Venkatadass VS (2008) The persistence of memory: contiguity effects across hundreds of seconds. Psychonom Bull Rev 15:58-63.

Howard MW, Eichenbaum H (2013) The hippocampus, time, and memory across scales. J Exp Psychol Gen 142:1211-1230.

Hsiao S, Isaacson RL (1971) Learning of food and water positions by hippocampus damaged rats. Physiol Behav 6:81-83.

Hsieh LT, Gruber MJ, Jenkins LJ, Ranganath C (2014) Hippocampal activity patterns carry information about objects in temporal context. Neuron 81:1165-1178.

Jenkins LJ, Ranganath C (2016) Distinct neural mechanisms for remembering when an event occurred. Hippocampus 26:554-559.

Kahana MJ (1996) Associative retrieval processes in free recall. Mem Cogn 24:103-109.

Keene CS, Bladon J, McKenzie S, Liu CD, O’Keefe J, Eichenbaum H (2016) Complementary functional organization of neuronal activity patterns in the perirhinal, lateral entorhinal, and medial entorhinal cortices. J Neurosci 36:3660-3675.

Kesner RP, Crutcher K, Beers DR (1988) Serial position curves for item (spatial location) information: role of the dorsal hippocampal formation and medial septum. Brain Res 454:219-226.

Kimble DP, Kimble RJ (1965) Hippocampectomy and response perseveration in the rat. J Comp Physiol Psychol 60:474-476.

Kobayashi T, Nishijo H, Fukuda M, Bures J, Ono T (1997) Task-dependent representations in rat hippocampal place neurons. J Neurophysiol 78:597-613.

Komorowski RW, Manns JR, Eichenbaum H (2009) Robust conjunctive item-place coding by hippocampal neurons parallels learning what happens where. J Neurosci 29:9918-9929.

Komorowski RW, Garcia CG, Wilson A, Hattori S, Howard MW, Eichenbaum H (2013) Ventral hippocampal neurons are shaped by experience to represent behaviorally relevant contexts. J Neurosci 33:8079-8087.

Leutgeb S, Leutgeb JK, Moser MB, Moser EI (2005) Place cells, spatial maps and the population code for memory. Curr Opin Neurobiol 15:738-746.

Liang F, Paulo R, Molina G, Clyde MA, Berger JO (2008) Mixtures of g priors for Bayesian variable selection. J Am Stat Assoc 103:410-423.

Mack ML, Love BC, Preston AR (2016) Dynamic updating of hippocampal object representations reflects new conceptual knowledge. Proc Natl Acad Sci U S A 113:13203-13208.

Mankin EA, Sparks FT, Slayyeh B, Sutherland RJ, Leutgeb S, Leutgeb JK (2012) Neuronal code for extended time in the hippocampus. Proc Natl Acad Sci U S A 109:19462-19467.

Mankin EA, Diehl GW, Sparks FT, Leutgeb S, Leutgeb JK (2015) Hippocampal CA2 activity patterns change over time to a larger extent than between spatial contexts. Neuron 85:190-201.

Manning JR, Polyn SM, Baltuch GH, Litt B, Kahana MJ (2011) Oscillatory patterns in temporal lobe reveal context reinstatement during memory search. Proc Natl Acad Sci U S A 108:12893-12897.

Manns JR, Howard MW, Eichenbaum H (2007) Gradual changes in hippocampal activity support remembering the order of events. Neuron 56: $530-540$

Markus EJ, Barnes CA, McNaughton BL, Gladden VL, Skaggs WE (1994) Spatial information content and reliability of hippocampal CA1 neurons: effects of visual input. Hippocampus 4:410-421.

Markus EJ, Qin YL, Leonard B, Skaggs WE, McNaughton BL, Barnes CA (1995) Interactions between location and task affect the spatial and directional firing of hippocampal neurons. J Neurosci 15:7079-7094.

Mau W, Sullivan DW, Kinsky NR, Hasselmo ME, Howard MW, Eichenbaum H (2018) The same hippocampal CA1 population simultaneously codes temporal information over multiple timescales. Curr Biol 28:1499_ 1508.e4.

McKenzie S, Frank AJ, Kinsky NR, Porter B, Rivière PD, Eichenbaum H (2014) Hippocampal representation of related and opposing memories develop within distinct, hierarchically organized neural schemas. Neuron 83:202-215

McNaughton BL, Barnes CA, Gerrard JL, Gothard K, Jung MW, Knierim JJ, Kudrimoti H, Qin Y, Skaggs WE, Suster M,Weaver KL (1996) Deciphering the hippocampal polyglot: the hippocampus as a path integration system. J Exp Biol 199:173-185.

Mehta MR, Quirk MC, Wilson MA (2000) Experience-dependent asymmetric shape of hippocampal receptive fields. Neuron 25:707-715.

Monaco JD, Rao G, Roth ED, Knierim JJ (2014) Attentive scanning behavior drives one-trial potentiation of hippocampal place fields. Nat Neurosci 17:725-731.

Muller RU, Kubie JL (1987) The effects of changes in the environment on the spatial firing of hippocampal complex-spike cells. J Neurosci 7:19511968.

Nielson DM, Smith TA, Sreekumar V, Dennis S, Sederberg PB (2015) Human hippocampus represents space and time during retrieval of realworld memories. Proc Natl Acad Sci U S A 112:11078-11083.

O'Keefe J, Nadel L (1978) The hippocampus as a cognitive map (Intergovernmental Panel on Climate Change, ed), Vol 53. Cambridge: Cambridge UP.

Paz R, Gelbard-Sagiv H, Mukamel R, Harel M, Malach R, Fried I (2010) A neural substrate in the human hippocampus for linking successive events. Proc Natl Acad Sci U S A 107:6046-6051.

Place R, Farovik A, Brockmann M, Eichenbaum H (2016) Bidirectional prefrontal-hippocampal interactions support context-guided memory. Nat Neurosci 19:992-994.

Rubin A, Geva N, Sheintuch L, Ziv Y (2015) Hippocampal ensemble dynamics timestamp events in long-term memory. Elife 4:e12247.

Schapiro AC, Kustner LV, Turk-Browne NB (2012) Shaping of object representations in the human medial temporal lobe based on temporal regularities. Curr Biol 22:1622-1627.

Schapiro AC, Turk-Browne NB, Norman KA, Botvinick MM (2016) Statis- 
tical learning of temporal community structure in the hippocampus. Hippocampus $26: 3-8$.

Schmitzer-Torbert N, Jackson J, Henze D, Harris K, Redish AD (2005) Quantitative measures of cluster quality for use in extracellular recordings. Neuroscience 131:1-11.

Smith DM, Bulkin DA (2014) The form and function of hippocampal context representations. Neurosci Biobehav Rev 40:52-61.

Sols I, DuBrow S, Davachi L, Fuentemilla L (2017) Event boundaries trigger rapid memory reinstatement of the prior events to promote their representation in long-term memory. Curr Biol 27:3499-3504.e4.

Swallow KM, Barch DM, Head D, Maley CJ, Holder D, Zacks JM (2011) Changes in events alter how people remember recent information. J Cogn Neurosci 23:1052-1064.

Tsao A, Sugar J, Lu L, Wang C, Knierim JJ, Moser MB, Moser EI (2018) Integrating time from experience in the lateral entorhinal cortex. Nature 561:57-62.

Tulving E (1972) Episodic and semantic memory. In: Organization of memory (Tulving E, Donaldson W, eds). Oxford, England: Academic Press.
Tulving E, Madigan SA (1970) Memory and verbal learning. Annu Rev Psychol 21:437-484

Wills TJ, Lever C, Cacucci F, Burgess N, O'Keefe J (2005) Attractor dynamics in the hippocampal representation of the local environment. Science 308:873-876.

Zacks JM, Tversky B, Iyer G (2001) Perceiving, remembering, and communicating structure in events. J Exp Psychol Gen 130:29-58.

Zaremba JD, Diamantopoulou A, Danielson NB, Grosmark AD, Kaifosh PW, Bowler JC, Liao Z, Sparks FT, Gogos JA, Losonczy A (2017) Impaired hippocampal place cell dynamics in a mouse model of the 22q11.2 deletion. Nat Neurosci 20:1612-1623.

Zaromb FM, Howard MW, Dolan ED, Sirotin YB, Tully M, Wingfield A, Kahana MJ (2006) Temporal associations and prior-list intrusions in free recall. J Exp Psychol Learn Mem Cogn 32:792-804.

Ziv Y, Burns LD, Cocker ED, Hamel EO, Ghosh KK, Kitch LJ, El Gamal A,Schnitzer MJ (2013) Long-term dynamics of CA1 hippocampal place codes. Nat Neurosci 16:264-266. 